\title{
UNSTEADY MHD PLANE COUETTE-POISEUILLE FLOW OF FOURTH- GRADE FLUID WITH THERMAL RADIATION, CHEMICAL REACTION AND SUCTION EFFECTS
}

\author{
K.M. JOSEPH* and E. AYANKOP-ANDI \\ Department of Mathematical Sciences, Nigerian Defence Academy, Kaduna, NIGERIA \\ E-mail: kpop.moses@kasu.edu.ng \\ S.U. MOHAMMED \\ Department of Mechanical Engineering, Nigerian Defence Academy, Kaduna, NIGERIA
}

\begin{abstract}
This study investigates the unsteady MHD flow of a fourth-grade fluid in a horizontal parallel plates channel. The upper plate is oscillating and moving while the bottom plate is stationary. Solutions for momentum, energy and concentration equations are obtained by the He-Laplace scheme. This method was also used by Idowu and Sani [12] and there is agreement with our results. The effect of various flow parameters controlling the physical situation is discussed with the aid of graphs. Significant results from this study show that velocity and temperature fields increase with the increase in the thermal radiation parameter, while velocity and concentric fields decrease with an increase in the chemical reaction parameter. Furthermore, velocity, temperature and concentric fields decrease with an increase in the suction parameter. It is also interesting to note that when $S_{4}=0$, our results will be in complete agreement with Idowu and Sani [12] results. The results of this work are applicable to industrial processes such as polymer extrusion of dye, draining of plastic films etc.
\end{abstract}

Keywords: thermal radiation, MHD, chemical reaction, fourth-grade fluid, suction, He-Laplace

\section{Introduction}

A fourth-grade fluid is an important subclass of differential type that is capable of describing shear thinning and shear thickening effects (examples are ketchup, blood, paint, cream, nail polish, etc.). This sort of model is used to explain the flow behaviour of non-Newtonian fluids which are considered vital and applicable in many industrial production processes such in the drilling of oil and gas wells, polymer extrusion from dye, glass fibre, paper production and draining of plastics films, etc.

Many empirical and semi-empirical non-Newtonian models or constitutive equations have been proposed. Rehan et al. [1] considered the steady flow of a fourth grade fluid between two parallel plates. They analyzed four types of flows: Couette flow, plug flow, Poiseuille flow and generalized Couette flow. The nonlinear differential equation describing the velocity field was solved by the optimal homotopy asymptotic method (OHAM). They observed that the OHAM was more efficient and flexible than the perturbation and homotopy analyses method. Islam et al. [2] considered the steady flow of a non-Newtonian fluid with slippage between the plate and the fluid. The constitutive equations of the fluids were modelled for a fourth-grade nonNewtonian fluid with partial slip. They employed homotopy perturbation and optimal homotopy asymptotic methods to solve the non-linear differential equation. Shehzad et al. [3] studied the electro-osmotic CouettePoiseuille flow of a power law A12O3- PVC nanofluid through a channel, in which the upper wall is moving with constant velocity. The influences of the magnetic field, mixed convection, Joule heating, and viscous dissipation were also examined. The flow was generated because of a constant pressure gradient in the axial direction. The resulting flow problem was described by coupled nonlinear ordinary differential equations,

\footnotetext{
${ }^{*}$ To whom correspondence should be addressed
} 
which were at first modeled and then transform into a dimensionless form through appropriate transformations. An analytical solution of the governing equation was given.

Fenuga et al. [4] investigated the mathematical model and solution for an unsteady MHD fourth grade fluid flow over a vertical plate in a porous medium with the effects of the magnetic field and suction/injection parameters using the Homotopy Perturbation Method. They displayed graphically and discussed the impact of dimensionless second, third and fourth grade parameters with the effects of the magnetic field and suction/injection parameters on the velocity field. They found out that on increase in the suction parameter decreases the momentum boundary layer thickness while the injection parameter enhances velocity distribution in the boundary layer. Magnetic field reduces velocity throughout the boundary layer because the Lorentz force which acts as a retarding force reduces the boundary layer thickness. Khan et al. [5] discussed the unsteady flow of a non- Newtonian fluid with the properties of heat/sink in the presence of thermal radiation. Santhosha et al. [6] studied the radiation and chemical combined effects on an MHD free convective heat and mass transfer flow of a viscous, incompressible, conducting elastic fluid through a porous medium limited by a porous plate within the presence of heat generation. The momentum, energy and mass diffusion equations were coupled non-linear partial differential equations. They employed the two term perturbation method.

Yurusoy [7] investigated the time dependent boundary layer flow of a modified power-law fluid of fourth grade on a stretched surface with an injection or suction boundary condition. The fluid model is a mixture of fourth grade and power-law fluids in which the fluid may display shear thickening, shear thinning or normal stress textures. He used the scaling and translation transformations which is a type of Lie Group transformation. Time dependent boundary layer equations were reduced into two alternative ordinary differential equations systems (ODEs) with boundary conditions. He found out that the boundary layer thickness decreases as the power-law index value increases. And also, as the fourth-grade fluid parameter, increases, the boundary layer thickness decreases while the velocity in the $y$ direction increases.

Taza et al. [8] studied the unsteady thin film flow of a fourth grade fluid over a moving and oscillating vertical belt. They employed the adomian decomposition method (ADM) and optimal homotopy asymptotic method (OHAM) to find the solution of the non- linear differential equations that governed the flow. Hayat et al. [9] presented the exact solution four four types of flows between two parallel plates, viz. Couette flow, plug flow, Poiseuille flow and generalized Couette flow. The nonlinear second-order differential equation for the velocity field was solved exactly in each case. The nonlinear differential equation describing the velocity field was solved by the optimal homotopy asymptotic method (OHAM). They observed that the OHAM is more efficient and flexible than the perturbation and homotopy analyses method. Arifuzzaman et al. [10] analysed heat and mass transfer characteristics of naturally corrective hydro-magnetic flows of a fourth grade radiative fluid flow throush a vertical porous plate. They considered a non-linear order chemical reaction and heat generation with thermal diffusion. The complete fundamental equations were transformed into dimensionless equations by implementing the finite difference scheme explicitly.

Priyadarsan and Panda [11] carried out a numerical investigation to study the unsteady flow of an incompressible and electrically conducting fourth-grade fluid through a porous medium between two infinite parallel plates under a transverse magnetic field with time-dependent suction. The lower plate is at rest and the upper plate is moving and oscillating in its own plane at about a constant mean velocity with time-dependent suction. The basic equations governing the flow and heat transfer are reduced to a set of non-linear partial differential equations. The governing equations are simplified using the perturbation method with respect to time and the resulting sixth-order non-linear differential equations are solved numerically using the RungeKutta method in association with the multi-shooting technique. Their investigation revealed that the highergrade fluid parameters influence significantly the fluid temperature.

Idowu and Sani [12] carried out an analysis for an unsteady magnetohydrodynamic (MHD) flow of a generalized third grade fluid between two parallel plates. The fluid flow was a result of the plate oscillation, movingment and pressure gradient. Three flow problems were investigated, namely: Couette, Poiseuille and Couette-Poiseuille flows and a number of nonlinear partial differential equations were obtained which were solved using the He-Laplace method. Expressions for the velocity field, temperature and concentration fields were given for each case and finally, effects of physical parameters on the fluid motion, temperature and concentration were plotted and discussed. They found that an increase in the thermal radiation parameter 
increases the temperature of the fluid and hence reduces the viscosity of the fluid while the concentration of the fluid reduces as the chemical reaction parameter increases.

In the above aforementioned investigations however, the effects of suction on the unsteady MHD flow of fourth-grade fluid with thermal radiation and chemical reaction have not been studied. Suction is the act or process of sucking. A force that causes a fluid or solid to be drawn into an interior space or to adhere to the surface because of the difference between the external and internal pressure. This is considered to be due to the porosity of the channel plates.

\section{Formulation of the problem}

We consider the unsteady flow of an electrically conducting incompressible fourth grade fluid between two horizontal parallel plates channel as shown in Fig.1. below. The fluid is subjected to a uniform transverse magnetic field. We assumed the bottom plate is fixed (stationary) and the top plate is moving with constant velocity.

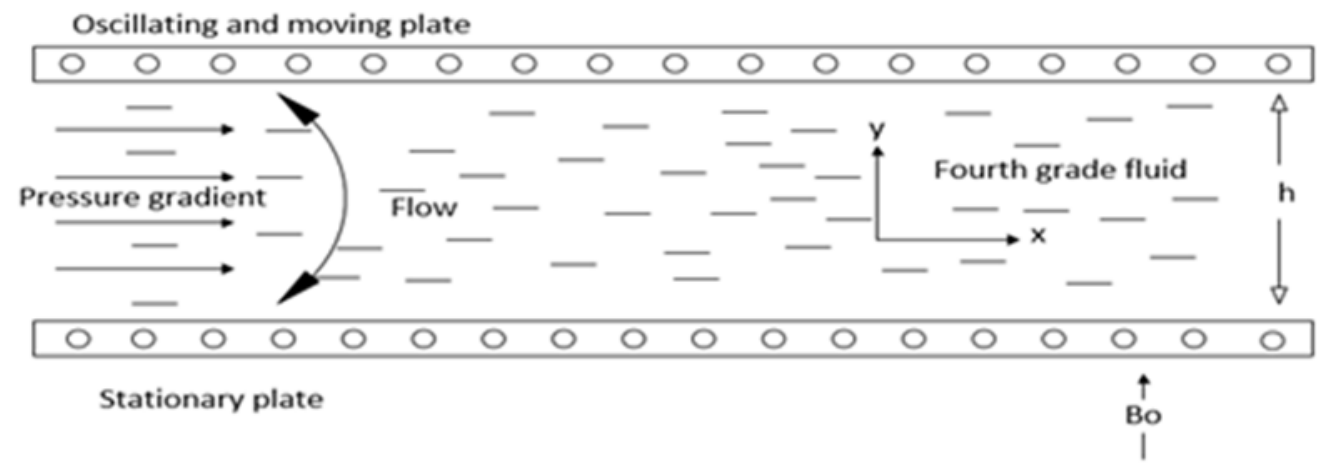

Fig.1. Physical configuration of the flow.

The state of this fluid is determined by the history of the deformation gradient without a preferred reference configuration. Its constitute equation can be written as

$$
T(x, t)=-P I+f_{s=0}^{\infty}\left(F_{t}^{t}(s)\right)
$$

where $P I$ is the undetermined part of the stress tensor, $F$ is the deformation gradient and $f$ is the functional. Coleman and Noll [13] studied a different sort of incompressible fluid grade $n$ as viscous fluid described in Hayat et al. [14]. An incompressible fluid of differential type of grade $n$ is a simple fluid obeying the constitutive equation

$$
T=-P I+\sum_{j=1}^{n} S_{j}
$$

obtained by asymptotic expansion of the functional in Eq.(2.1) through a retardation parameter $\alpha$. For $n=4$ as in Hayat et al. ([14], [15]]) and Arifuzzaman et al. [10], the first four (4) tensors $S_{j}$ are given by

$$
\begin{aligned}
& S_{1}=\mu A_{1}, \\
& S_{2}=\alpha_{1} A_{2}+\alpha_{2} A_{1}^{2},
\end{aligned}
$$




$$
\begin{aligned}
& S_{3}=\beta_{1} A_{3}+\beta_{2}\left(A_{1} A_{2}+A_{2} A_{1}\right)+\beta_{3}\left(\operatorname{tr} A_{1}^{2}\right) A_{1}, \\
& S_{4}=\gamma_{1} A_{4}+\gamma_{2}\left(A_{3} A_{1}+A_{1} A_{3}\right)+\gamma_{3} A_{2}^{2}+\gamma_{4}\left(A_{2} A_{1}^{2}+A_{1}^{2} A_{2}\right)+ \\
& +\gamma_{5}\left(t r A_{2}\right) A_{2}+\gamma_{6}\left(\operatorname{tr} A_{2}\right) A_{1}^{2}+\left[\gamma_{7} \operatorname{tr} A_{3}+\gamma_{8} \operatorname{tr}\left(A_{2} A_{1}\right)\right] A_{1}
\end{aligned}
$$

where, $\mu$ is the coefficient of shear viscosity, $\alpha_{i}(i=1,2), \beta_{i}(i=1,2,3)$ and $\gamma_{i}(i=1(1) 8)$ are material constants. The $A_{n}$ are the Rivlin-Ericksen tensors defined by the recursion relation

$$
\begin{aligned}
& A_{n}=\frac{d}{d t} A_{n-1}+A_{n-1} L+L^{T} A_{n-1, n>1}, \\
& A_{1}=L+L^{T}
\end{aligned}
$$

where $L=\nabla V, \frac{d}{d t}$ is the material time derivative and $V$ is the velocity.

We note that when $\gamma_{i}=0$, the fourth grade model reduces to the third grade model. When $\beta_{i}=0$, the third grade model reduces to a second grade model. When $\alpha_{i}=0, \beta_{i}=0$ and $\gamma_{i}=0$ then the model reduces to classical Navier-Stoke fluid.

The thermally radiative and chemically reactive flow is heading towards the $x$-direction along infinite porous plate with heat generation. Here, $U_{0}$ is the uniform velocity, $T_{\infty}$ and $C_{\infty}$ are the fluid temperature and concentration.

Under the above assumption, the equations that described the physical circumstances are

$$
\begin{aligned}
& \frac{\partial \mathrm{v}}{\partial y}=0 \\
& \frac{\partial u}{\partial t}+\mathrm{v} \frac{\partial u}{\partial y}=-\frac{\partial p}{\partial x}+v \frac{\partial^{2} u}{\partial y^{2}}+\frac{\alpha_{1} v}{\rho} \frac{\partial^{3} u}{\partial y^{2} \partial t}+\frac{\beta_{1} v^{2}}{\rho} \frac{\partial^{4} u}{\partial y^{2} \partial t^{2}}+\frac{\sigma\left(\beta_{2}+\beta_{3}\right)}{\rho}\left(\frac{\partial u}{\partial y}\right)^{2} \frac{\partial^{2} u}{\partial y^{2}}+ \\
& +\frac{\gamma_{1} v^{3}}{\rho} \frac{\partial^{5} u}{\partial y^{2} \partial t^{3}}+\frac{2 v\left(3 \gamma_{2}+\gamma_{3}+\gamma_{4}+\gamma_{5}+\gamma_{7}+\gamma_{8}\right)}{\rho C_{p}}\left[2 \frac{\partial u}{\partial y} \frac{\partial^{2} u}{\partial y^{2}} \frac{\partial^{2} u}{\partial t \partial y}+\left(\frac{\partial u}{\partial y}\right)^{2} \frac{\partial^{3} u}{\partial y^{2} \partial t}\right]+ \\
& -\frac{\sigma B_{0}^{2}}{\rho C_{p}} u+g \beta_{T}\left(T-T_{\infty}\right)+g \beta_{C}\left(C-C_{\infty}\right)-\frac{v}{k} u, \\
& \frac{\partial T}{\partial t}+\mathrm{v} \frac{\partial T}{\partial y}=\frac{k}{\rho C_{p}} \frac{\partial^{2} T}{\partial y^{2}}+\frac{Q_{0}}{\rho C_{p}}\left(T_{w}-T_{\infty}\right)-\frac{1}{\rho C_{p}} \frac{\partial q_{r}}{\partial y} \\
& \frac{\partial C}{\partial t}+\mathrm{v} \frac{\partial C}{\partial y}=D \frac{\partial^{2} C}{\partial y^{2}}-K_{c}\left(C-C_{\infty}\right) .
\end{aligned}
$$

From Eq.(2.11), $q_{r}$ is the radiative heat flux defined as 


$$
\frac{\partial q_{r}}{\partial y}=4 \alpha^{2}\left(T_{w}-T_{\infty}\right)
$$

The initial and boundary conditions are

$$
\begin{aligned}
& u=U_{0} e^{-y h}, \quad T=T_{0}+\left(T_{w}-T_{\infty}\right) e^{-y h}, C=C_{0}+\left(C_{w}-C_{\infty}\right) e^{-y h} \text { at } t=0 \text { for } 0 \leq y \geq h, \\
& u(y, t)=U, \quad T(y, t)=T_{w}, \quad C(y, t)=C_{w} \quad \text { at } \quad y=h \quad \text { for } \quad t \geq 0, \\
& u(y, t) \rightarrow \infty, \quad T(y, t) \rightarrow \infty, \quad C(y, t) \rightarrow \infty \quad \text { as } \quad y \rightarrow \infty \quad \text { for } \quad t>0 .
\end{aligned}
$$

where $u$ is the fluid velocity, $T$ is the temperature and $C$ is the species concentration equation, $q_{r}$ is the radiative heat flux, $\rho$ is the density of the fluid, $C_{p}$ is the heat capacity, $B_{0}$ is the external magnetic field. In order to transform Eqs (2.10)-(2.13), we use the following dimensionless parameters

$$
\begin{aligned}
& u^{*}=\frac{u}{U_{0}}, \quad p^{*}=\frac{p}{\mu U_{0}^{2}}, \quad t^{*}=\frac{t U_{0}^{2}}{v}, \quad H a^{2}=\frac{\sigma B_{0}^{2} v}{\rho U_{0}^{2}}, \quad G_{r}=\frac{g \beta_{T}\left(T_{w}-T_{\infty}\right) v}{U_{0}^{3}}, \\
& G_{c}=\frac{g \beta_{C}\left(C_{w}-C_{\infty}\right) v}{U_{0}^{3}}, \quad D a=\frac{K U_{0}^{2}}{h^{2}}, \quad S_{c}=\frac{D}{v}, \quad y^{*}=\frac{y U_{0}}{v}, \quad x^{*}=\frac{x}{h}, \\
& h=\frac{U_{0}}{v}, \quad P_{r}=\frac{k U_{0}^{2}}{v^{2}}, \quad S=\frac{\mathrm{v}_{0}}{U_{0}}, \quad \mathrm{v}=\frac{\mathrm{v}}{U_{0}}, \quad \theta=\frac{T-T_{0}}{T_{w}-T_{\infty}}, \quad C^{*}=\frac{C-C_{0}}{C_{w}-C_{\infty}}, \\
& \delta=\frac{4 \alpha^{2} U_{0}^{2}}{\rho c_{p} v}, \quad \alpha=\frac{\alpha U_{0}^{2}}{\rho v^{2}}, \quad \beta_{a}=\frac{\beta_{1} U_{0}^{4}}{\rho v^{3}}, \quad \beta_{b}=\frac{\left(\beta_{2}+\beta_{3}\right) U_{0}^{4}}{\rho v^{3}}, \quad \gamma_{a}=\frac{\gamma_{1} U_{0}^{6}}{\rho v^{3}}, \\
& \gamma_{b}=\frac{2\left(3 \gamma_{2}+\gamma_{3}+\gamma_{4}+\gamma_{5}+3 \gamma_{7}+\gamma_{8}\right) U_{0}^{6}}{\rho v^{4}}, \quad D a=\frac{k U_{0}^{2}}{v^{2}}, \quad K_{r}=\frac{K_{c} v}{U_{0}^{2}} .
\end{aligned}
$$

Substituting Eq.(2.14) into Eq.(2.10)-(2.13) and by dropping the asterisks, we have the following:

$$
\begin{aligned}
& \frac{\partial v}{\partial y}=0 \quad \Rightarrow \quad \mathrm{v}=-\mathrm{v}_{0} \\
& \frac{\partial u}{\partial t}-S \frac{\partial u}{\partial y}=-\frac{\partial p}{\partial x}+\frac{\partial^{2} u}{\partial y^{2}}+\alpha \frac{\partial^{3} u}{\partial y^{2} \partial t}+\beta_{a} \frac{\partial^{4} u}{\partial y^{2} \partial t^{2}}+\beta_{b}\left(\frac{\partial u}{\partial y}\right)^{2} \frac{\partial^{2} u}{\partial y^{2}}+\gamma_{a} \frac{\partial^{5} u}{\partial y^{2} \partial t^{3}}+ \\
& +\gamma_{b}\left[2 \frac{\partial u}{\partial y} \frac{\partial^{2} u}{\partial y^{2}} \frac{\partial^{2} u}{\partial t \partial y}+\left(\frac{\partial u}{\partial y}\right)^{2} \frac{\partial^{3} u}{\partial y^{2} \partial t}\right]-\left(H a+\frac{1}{D a}\right) u+G_{r} \theta+G_{c} C
\end{aligned}
$$




$$
\begin{aligned}
& \frac{\partial \theta}{\partial t}-S \frac{\partial \theta}{\partial y}=\frac{1}{P_{r}} \frac{\partial^{2} \theta}{\partial y^{2}}+\left(Q_{0}+\delta\right) \theta \\
& \frac{\partial C}{\partial t}-S \frac{\partial C}{\partial y}=\frac{1}{S_{c}} \frac{\partial^{2} C}{\partial y^{2}}-K_{r} C
\end{aligned}
$$

And the initial and boundary conditions become

$$
\begin{aligned}
& \left.u(y, t)=e^{-y}, \quad \theta(y, t)=e^{-y}, \quad C(y, t)=e^{-y} \quad \text { at } \quad t=0 \quad \text { for } \quad 0 \leq y \geq 1\right) \\
& u(y, t)=1, \quad \theta(y, t)=1, \quad C(y, t)=1 \quad \text { at } \quad y=1 \quad \text { for } \quad t \geq 0, \\
& u(y, t) \rightarrow \infty, \quad T(y, t) \rightarrow \infty, \quad C(y, t) \rightarrow \infty \quad \text { as } \quad y \rightarrow \infty \text { for } t>0 .
\end{aligned}
$$

\section{Method of solution of the problem}

In this section we employed the He-Laplace scheme to solve Eq.(2.16) to (2.18) subjected to the initial and boundary conditions (2.19).

Since Eq.(2.16) is a coupled non-linear partial differential equation, we have to solve Eq.(2.17) and (2.18) first.

Now applying Laplace transform on Eq.(2.18), we have

$$
L\left\{\frac{\partial C}{\partial t}\right\}-S L\left\{\frac{\partial C}{\partial y}\right\}=\frac{1}{S_{c}} L\left\{\frac{\partial^{2} C}{\partial y^{2}}\right\}-L\left\{K_{r} C\right\} .
$$

Applying the initial condition and dividing through by $s$ and rearranging, we obtain

$$
L\{C(y, t)\}=\frac{e^{-y}}{s}+\frac{1}{s}\left\{\frac{1}{S_{c}} L\left\{\frac{\partial^{2} C}{\partial y^{2}}\right\}+S L\left\{\frac{\partial C}{\partial y}\right\}-L\left\{K_{r} C\right\}\right\} .
$$

Taking the inverse Laplace transform of both sides of Eq.(3.2), gives

$$
C(y, t)=e^{-y}+L^{-1}\left[\frac{1}{S}\left\{\frac{1}{S_{c}} L\left\{\frac{\partial^{2} C}{\partial y^{2}}\right\}+S L\left\{\frac{\partial C}{\partial y}\right\}-L\left\{K_{r} C\right\}\right\}\right]
$$

Applying the homotopy perturbation technique, Eq.(3.3) yields

$$
\sum_{n=0}^{\infty} P^{n} C_{n}(y, t)=e^{-y}+P\left[L^{-1}\left\{\frac{1}{s}\left\{\frac{1}{S_{c}} L\left\{\frac{\partial^{2} C}{\partial y^{2}}\right\}-L\left\{K_{r} C\right\}\right\}\right\}\right] .
$$

Comparing the coefficients of the like powers of ' $P$ ', the following approximations were obtained

$$
P^{0}: C_{0}(y, t)=e^{-y}
$$




$$
\begin{aligned}
& P^{l}: C_{1}(y, t)=L^{-1}\left[\frac{1}{S}\left\{\frac{1}{S_{c}} L\left\{\frac{\partial^{2} C_{0}}{\partial y^{2}}\right\}+S L\left\{\frac{\partial C_{0}}{\partial y}\right\}-L\left\{K_{r} C_{0}\right\}\right\}\right]= \\
& =L^{-1}\left\{\frac{1}{S_{c}}\left(\frac{e^{-y}}{s^{2}}\right)-S\left(\frac{e^{-y}}{s^{2}}\right)-K_{r}\left(\frac{e^{-y}}{s^{2}}\right)\right\}=\left(\frac{e^{-y}}{S_{c}}-S e^{-y}-K_{r} e^{-y}\right) t, \\
& P^{2}: C_{2}(y, t)=L^{-1}\left[\frac{1}{S}\left\{\frac{1}{S_{c}} L\left\{\frac{\partial^{2} C_{1}}{\partial y^{2}}\right\}+S L\left\{\frac{\partial C_{1}}{\partial y}\right\}-L\left\{K_{r} C_{1}\right\}\right\}\right]= \\
& =L^{-1}\left[\frac { 1 } { S } \left\{\frac{1}{S_{c}} L\left\{\left(\frac{e^{-y}}{S_{c}}-S e^{-y}-K_{r} e^{-y}\right) t\right\}-S L\left\{\left(S e^{-y}-\frac{e^{-y}}{S_{c}}+K_{r} e^{-y}\right) t\right\}[\{+\}]=\right.\right. \\
& \left.\left.-L\left\{K_{r}\left(\frac{e^{-y}}{S_{c}}-S e^{-y}-K_{r} e^{-y}\right) t\right\}\right\}\right]=\left(\frac{e^{-y}}{S_{c}^{2}}-\frac{2 S e^{-y}}{S_{c}}-\frac{2 K_{r} e^{-y}}{S_{c}}+\right. \\
& \left.+2 K_{r} S e^{-y}+S^{2} e^{-y}+K_{r}^{2} e^{-y}\right) \frac{t^{2}}{2 !}, \\
& P^{3}: C_{3}(y, t)=L^{-1}\left[\frac{1}{s}\left\{\frac{1}{S_{c}} L\left\{\frac{\partial^{2} C_{2}}{\partial y^{2}}\right\}+S L\left\{\frac{\partial C_{2}}{\partial y}\right\}-L\left\{K_{r} C_{2}\right\}\right\}\right]= \\
& =L^{-1}\left[\frac { 1 } { s } \left\{\frac{1}{S_{c}} L\left\{\left(\frac{e^{-y}}{S_{c}^{2}}-\frac{2 S e^{-y}}{S_{c}}-\frac{2 K_{r} e^{-y}}{S_{c}}++2 K_{r} S e^{-y}+S^{2} e^{-y}+K_{r}^{2} e^{-y}\right) \frac{t^{2}}{2 !}\right\}+\right.\right. \\
& +S L\left\{\left(\frac{e^{-y}}{S_{c}^{2}}-\frac{2 S e^{-y}}{S_{c}}-\frac{2 K_{r} e^{-y}}{S_{c}}+2 K_{r} S e^{-y}+S^{2} e^{-y}+K_{r}^{2} e^{-y}\right) \frac{t^{2}}{2 !}\right\}+ \\
& \left.-L\left\{K_{r}\left(\frac{e^{-y}}{S_{c}^{2}}-\frac{2 S e^{-y}}{S_{c}}-\frac{2 K_{r} e^{-y}}{S_{c}}++2 K_{r} S e^{-y}+S^{2} e^{-y}+K_{r}^{2} e^{-y}\right) \frac{t^{2}}{2 !}\right\}\right\}= \\
& =\left(\frac{e^{-y}}{S_{c}^{3}}-\frac{3 S e^{-y}}{S_{c}^{2}}-\frac{2 K_{r} e^{-y}}{S_{c}^{2}}+\frac{6 K_{r} S e^{-y}}{S_{c}}+\frac{3 S^{2} e^{-y}}{S_{c}}+\frac{3 K_{r}^{2} e^{-y}}{S_{c}}-\frac{K_{r} e^{-y}}{S_{c}}+\right. \\
& \left.-K_{r} S^{2} e^{-y}-3 K_{r}^{2} S e^{-y}-S^{3} e^{-y}-K_{r}^{3} e^{-y}\right) \frac{t^{3}}{3 !} \text {. }
\end{aligned}
$$

Therefore, in view of equations (3.5), (3.6), (3.7) and (3.8), the solution to equation (2.18) is

$$
\begin{aligned}
& C(y, t)=C_{0}(y, t)+C_{1}(y, t)+C_{2}(y, t)+C_{3}(y, t) \cdots \\
& C(y, t)=e^{-y}+\left(\frac{e^{-y}}{S_{c}}-S e^{-y}-K_{r} e^{-y}\right) t+\left(\frac{e^{-y}}{S_{c}^{2}}-\frac{2 S e^{-y}}{S_{c}}-\frac{2 K_{r} e^{-y}}{S_{c}}+2 K_{r} S e^{-y}+\right. \\
& \left.+S^{2} e^{-y}+K_{r}^{2} e^{-y}\right) \frac{t^{2}}{2 !}+\left(\frac{e^{-y}}{S_{c}^{3}}-\frac{3 S e^{-y}}{S_{c}^{2}}-\frac{2 K_{r} e^{-y}}{S_{c}^{2}}+\frac{6 K_{r} S e^{-y}}{S_{c}}+\frac{3 S^{2} e^{-y}}{S_{c}}+\right.
\end{aligned}
$$




$$
\left.+\frac{3 K_{r}^{2} e^{-y}}{S_{c}}-\frac{K_{r} e^{-y}}{S_{c}}-K_{r} S^{2} e^{-y}-3 K_{r}^{2} S e^{-y}-S^{3} e^{-y}-K_{r}^{3} e^{-y}\right) \frac{t^{3}}{3 !}+\cdots
$$

Next, we consider Eq.(2.17), which is rearranged to give

$$
\frac{\partial \theta}{\partial t}-S \frac{\partial \theta}{\partial y}=\frac{1}{P_{r}} \frac{\partial^{2} \theta}{\partial y^{2}}+\left(l_{l}\right) \theta \quad \text { where } \quad l_{l}=Q_{0}+\delta .
$$

Now applying the Laplace transform to Eq.(2.16)

$$
L\left\{\frac{\partial \theta}{\partial t}\right\}-L\left\{S \frac{\partial \theta}{\partial y}\right\}=\frac{1}{P_{r}} L\left\{\frac{\partial^{2} \theta}{\partial y^{2}}\right\}+L\left\{l_{l} \theta\right\} .
$$

Applying the initial condition and dividing by $s$ and rearranging we obtain

$$
L\{\theta(y, t)\}=\frac{e^{-y}}{s}+\frac{1}{s}\left\{\frac{1}{P_{r}} L\left\{\frac{\partial^{2} \theta}{\partial y^{2}}\right\}+S L\left\{\frac{\partial \theta}{\partial y}\right\}+L\left\{l_{l} \theta\right\}\right\} .
$$

Taking the inverse Laplace transform of both sides of Eq.(31) gives

$$
\theta(y, t)=e^{-y}+L^{-1}\left[\frac{1}{S}\left\{\frac{1}{P_{r}} L\left\{\frac{\partial^{2} \theta}{\partial y^{2}}\right\}+S L\left\{\frac{\partial \theta}{\partial y}\right\}+L\left\{l_{l} \theta\right\}\right\}\right] .
$$

Applying the homotopy perturbation technique to Eq.(3.13), yields

$$
\sum_{n=0}^{\infty} P^{n} \theta_{n}(y, t)=e^{-y}+P\left[L^{-1}\left\{\frac{1}{s}\left\{\frac{1}{P_{r}} L\left\{\frac{\partial^{2} \theta}{\partial y^{2}}\right\}+L\{\delta \theta\}\right\}\right\} .\right.
$$

Comparing the coefficients of the like powers of ' $P$ ' in Eq.(3.14), the following approximations are obtained

$$
\begin{aligned}
& P^{0}: \theta_{0}(y, t)=e^{-y}, \\
& P^{l}: \theta_{l}(y, t)=L^{-1}\left[\frac{1}{s}\left\{\frac{1}{P_{r}} L\left\{\frac{\partial^{2} \theta_{0}}{\partial y^{2}}\right\}+S L\left\{\frac{\partial \theta_{0}}{\partial y}\right\}+L\left\{l_{l} \theta_{0}\right\}\right\}\right]= \\
& =L^{-1}\left\{\frac{1}{P_{r}}\left(\frac{e^{-y}}{s^{2}}\right)-S\left(\frac{e^{-y}}{s^{2}}\right)+l_{l}\left(\frac{e^{-y}}{s^{2}}\right)\right\}=\left(\frac{e^{-y}}{P_{r}}-S e^{-y}+l_{l} e^{-y}\right) t, \\
& P^{2}: \theta_{2}(y, t)=L^{-1}\left[\frac{1}{S}\left\{\frac{1}{P_{r}} L\left\{\frac{\partial^{2} \theta_{l}}{\partial y^{2}}\right\}+S L\left\{\frac{\partial \theta_{1}}{\partial y}\right\}+L\left\{l_{l} \theta_{l}\right\}\right\}\right]= \\
& =L^{-1}\left[\left\{\frac{1}{S} \frac{1}{P_{r}} L\left\{\left(\frac{e^{-y}}{P_{r}}-S e^{-y}+l_{l} e^{-y}\right) t\right\}-S L\left\{\left(S e^{-y}-\frac{e^{-y}}{P_{r}}-l_{l} e^{-y}\right) t\right\}+\right.\right. \\
& \left.+L\left\{l_{1}\left(\frac{e^{-y}}{P_{r}}-S e^{-y}+l_{l} e^{-y}\right) t\right\}\right\}=\left(\frac{e^{-y}}{P_{r}^{2}}+\frac{2 l_{1} e^{-y}}{P_{r}}-S^{2} e^{-y}+l_{l}^{2} e^{-y}\right) \frac{t^{2}}{2 !},
\end{aligned}
$$




$$
\begin{aligned}
& P^{3}: \theta_{3}(y, t)=L^{-1}\left[\frac{1}{S}\left\{\frac{1}{P_{r}} L\left\{\frac{\partial^{2} \theta_{2}}{\partial y^{2}}\right\}+S L\left\{\frac{\partial \theta_{2}}{\partial y}\right\}+L\left\{l_{l} \theta_{2}\right\}\right\}=\right. \\
& =L^{-1}\left[\frac { 1 } { s } \left\{\frac{1}{P_{r}} L\left\{\left(\frac{e^{-y}}{P_{r}^{2}}+\frac{2 l_{1} e^{-y}}{P_{r}}-S^{2} e^{-y}+l_{1}^{2} e^{-y}\right) \frac{t^{2}}{2 !}\right\}-S L\left\{\left(\frac{e^{-y}}{P_{r}^{2}}+\frac{2 l_{1} e^{-y}}{P_{r}}+\right.\right.\right.\right. \\
& \left.\left.\left.\left.-S^{2} e^{-y}+l_{1}^{2} e^{-y}\right) \frac{t^{2}}{2 !}\right\}++\left(S^{2} e^{-y}-\frac{e^{-y}}{P_{r}^{2}}-\frac{2 l_{l} e^{-y}}{P_{r}}-l_{1}^{2} e^{-y}\right) \frac{t^{2}}{2 !}\right\}\right]= \\
& =\left(\frac{e^{-y}}{P_{r}^{3}}+\frac{3 l_{1} e^{-y}}{P_{r}^{2}}-\frac{S^{2} e^{-y}}{P_{r}}+\frac{3 l_{1}^{2} e^{-y}}{P_{r}}-\frac{S e^{-y}}{P_{r}}-\frac{2 l_{1} S e^{-y}}{P_{r}}+\right. \\
& \left.-l_{1}^{2} S e^{-y}-l_{1} S^{2} e^{-y}+l_{l}^{3} e^{-y}+S^{3} e^{-y}\right) \frac{t^{3}}{3 !} .
\end{aligned}
$$

Therefore, in view of Eqs (3.15)-(3.18), the solution to (2.17) is,

$$
\begin{aligned}
& \theta(y, t)=\theta_{0}(y, t)+\theta_{1}(y, t)+\theta_{2}(y, t)+\theta_{3}(y, t) \cdots, \\
& \theta(y, t)=e^{-y}+\left(\frac{e^{-y}}{P_{r}}-S e^{-y}+l_{1} e^{-y}\right) t+\left(\frac{e^{-y}}{P_{r}^{2}}+\frac{2 l_{1} e^{-y}}{P_{r}}-S^{2} e^{-y}+l_{l}^{2} e^{-y}\right) \frac{t^{2}}{2 !}+ \\
& +\left(\frac{e^{-y}}{P_{r}^{3}}+\frac{3 l_{1} e^{-y}}{P_{r}^{2}}-\frac{S^{2} e^{-y}}{P_{r}}+\frac{3 l_{1}^{2} e^{-y}}{P_{r}}-\frac{S e^{-y}}{P_{r}}-\frac{2 l_{1} S e^{-y}}{P_{r}}+\right. \\
& \left.-l_{l}^{2} S e^{-y}-l_{1} S^{2} e^{-y}+l_{1}^{3} e^{-y}+S^{3} e^{-y}\right) \frac{t^{3}}{3 !}+\cdots .
\end{aligned}
$$

Finally, we now solve Eq.(2.16), which is rearranged to give

$$
\begin{aligned}
& \frac{\partial u}{\partial t}-S \frac{\partial u}{\partial y}=-\frac{\partial p}{\partial x}+\frac{\partial^{2} u}{\partial y^{2}}+\alpha \frac{\partial^{3} u}{\partial y^{2} \partial t}+\beta_{a} \frac{\partial^{4} u}{\partial y^{2} \partial t^{2}}+\beta_{b}\left(\frac{\partial u}{\partial y}\right)^{2} \frac{\partial^{2} u}{\partial y^{2}}+ \\
& +\gamma_{a} \frac{\partial^{5} u}{\partial y^{2} \partial t^{3}}+\gamma_{b}\left[2 \frac{\partial u}{\partial y} \frac{\partial^{2} u}{\partial y^{2}} \frac{\partial^{2} u}{\partial t \partial y}+\left(\frac{\partial u}{\partial y}\right)^{2} \frac{\partial^{3} u}{\partial y^{2} \partial t}\right]-l_{2} u+G_{r} \theta+G_{c} C
\end{aligned}
$$

where

$$
H a+\frac{1}{D a}=l_{2}
$$

Applying the Laplace transform to both sides of Eq.(3.19a) gives

$$
\begin{aligned}
& L\left\{\frac{\partial u}{\partial t}\right\}-L\left\{S \frac{\partial u}{\partial y}\right\}=L\left\{-\frac{\partial p}{\partial x}+\frac{\partial^{2} u}{\partial y^{2}}+\alpha \frac{\partial^{3} u}{\partial y^{2} \partial t}+\beta_{a} \frac{\partial^{4} u}{\partial y^{2} \partial t^{2}}+\beta_{b}\left(\frac{\partial u}{\partial y}\right)^{2} \frac{\partial^{2} u}{\partial y^{2}}+\right. \\
& \left.+\gamma_{a} \frac{\partial^{5} u}{\partial y^{2} \partial t^{3}}+\gamma_{b}\left[2 \frac{\partial u}{\partial y} \frac{\partial^{2} u}{\partial y^{2}} \frac{\partial^{2} u}{\partial t \partial y}+\left(\frac{\partial u}{\partial y}\right)^{2} \frac{\partial^{3} u}{\partial y^{2} \partial t}\right]-l_{2} u+G_{r} \theta+G_{c} C\right\} .
\end{aligned}
$$


but

$$
L\left\{\frac{\partial u}{\partial t}\right\}=s L\{u(y, t)\}-u(y, 0)
$$

Hence

$$
\begin{aligned}
& L\{u(y, t)\}=\frac{u(y, 0)}{s}+\frac{1}{s} L\left\{-\frac{\partial p}{\partial x}+S \frac{\partial u}{\partial y}+\frac{\partial^{2} u}{\partial y^{2}}+\alpha \frac{\partial^{3} u}{\partial y^{2} \partial t}+\beta_{a} \frac{\partial^{4} u}{\partial y^{2} \partial t^{2}}+\beta_{b}\left(\frac{\partial u}{\partial y}\right)^{2} \frac{\partial^{2} u}{\partial y^{2}}+\right. \\
& \left.+\gamma_{a} \frac{\partial^{5} u}{\partial y^{2} \partial t^{3}}+\gamma_{b}\left[2 \frac{\partial u}{\partial y} \frac{\partial^{2} u}{\partial y^{2}} \frac{\partial^{2} u}{\partial t \partial y}+\left(\frac{\partial u}{\partial y}\right)^{2} \frac{\partial^{3} u}{\partial y^{2} \partial t}\right]-l_{2} u+G_{r} \theta+G_{c} C\right\} .
\end{aligned}
$$

Taking the inverse Laplace transform of both sides of Eq.(3.22), we have

$$
\begin{aligned}
& L^{-1}\{L\{u(y, t)\}\}=L^{-1}\left\{\frac{u(y, 0)}{s}-\frac{\partial p}{\partial x}+\frac{1}{S} L\left\{S \frac{\partial u}{\partial y}+\frac{\partial^{2} u}{\partial y^{2}}+\alpha \frac{\partial^{3} u}{\partial y^{2} \partial t}+\beta_{\mathrm{a}} \frac{\partial^{4} u}{\partial y^{2} \partial t^{2}}+\right.\right. \\
& +\beta_{b}\left(\frac{\partial u}{\partial y}\right)^{2} \frac{\partial^{2} u}{\partial y^{2}}+\gamma_{\mathrm{a}} \frac{\partial^{5} u}{\partial y^{2} \partial t^{3}}+\gamma_{b}\left[2 \frac{\partial u}{\partial y} \frac{\partial^{2} u}{\partial y^{2}} \frac{\partial^{2} u}{\partial t \partial y}+\left(\frac{\partial u}{\partial y}\right)^{2} \frac{\partial^{3} u}{\partial y^{2} \partial t}\right]-l_{2} u+ \\
& +\frac{G_{r}}{S}\left(e^{-y}+\left(\frac{e^{-y}}{P_{r}}-S e^{-y}+l_{1} e^{-y}\right) t+\left(\frac{e^{-y}}{P_{r}^{2}}+\frac{2 l_{1} e^{-y}}{P_{r}}-S^{2} e^{-y}+l_{1}^{2} e^{-y}\right) \frac{t^{2}}{2 !}\left(\frac{t^{3}}{3 !}\right)+\right. \\
& +\left(\frac{e^{-y}}{P_{r}^{3}}+\frac{3 l_{1} e^{-y}}{P_{r}^{2}}-\frac{S^{2} e^{-y}}{P_{r}}+\frac{3 l_{1}^{2} e^{-y}}{P_{r}}-\frac{S e^{-y}}{P_{r}}-\frac{2 l_{1} S e^{-y}}{P_{r}}-l_{1}^{2} S e^{-y}-l_{1} S^{2} e^{-y}+\right. \\
& \left.\left.+l_{1}^{3} e^{-y}+S^{3} e^{-y}\right)\right)+\frac{G_{c}}{S}\left(e^{-y}+\left(\frac{e^{-y}}{S_{c}}-S e^{-y}-K_{r} e^{-y}\right) t+\left(\frac{e^{-y}}{S_{c}^{2}}-\frac{2 S e^{-y}}{S_{c}}+\right.\right. \\
& \left.-\frac{2 K_{r} e^{-y}}{S_{c}}++2 K_{r} S e^{-y}+S^{2} e^{-y}+K_{r}^{2} e^{-y}\right) \frac{t^{2}}{2 !}+\left(\frac{e^{-y}}{S_{c}^{3}}-\frac{3 S e^{-y}}{S_{c}^{2}}-\frac{2 K_{r} e^{-y}}{S_{c}^{2}}+\right. \\
& +\frac{6 K_{r} S e^{-y}}{S_{c}}+\frac{3 S^{2} e^{-y}}{S_{c}}+\frac{3 K_{r}^{2} e^{-y}}{S_{c}}+-\frac{K_{r} e^{-y}}{S_{c}}-K_{r} S^{2} e^{-y}-3 K_{r}^{2} S e^{-y}+ \\
& \left.\left.\left.\left.-S^{3} e^{-y}-K_{r}^{3} e^{-y}\right) \frac{t^{3}}{3 !}\right)\right\}\right\},
\end{aligned}
$$

or

$$
\begin{aligned}
& u(y, t)=\lambda+e^{-y}+\left(G_{c} e^{-y}+G_{r} e^{-y}\right) t+\left(\frac{e^{-y}}{P_{r}}-2 S e^{-y}+l_{1} e^{-y}+\frac{e^{-y}}{S_{c}}-K_{r} e^{-y}\right) \frac{t^{2}}{2 !}+ \\
& +\left(\frac{e^{-y}}{P_{r}^{2}}+\frac{2 l_{1} e^{-y}}{P_{r}}+l_{1}^{2} e^{-y}+\frac{e^{-y}}{S_{c}^{2}}-\frac{2 S e^{-y}}{S_{c}}-\frac{2 K_{r} e^{-y}}{S_{c}}+2 K_{r} S e^{-y}+K_{r}^{2} e^{-y}\right) \frac{t^{3}}{3 !}+ \\
& +\left(\frac{e^{-y}}{P_{r}^{3}}+\frac{3 l_{1} e^{-y}}{P_{r}^{2}}-\frac{S^{2} e^{-y}}{P_{r}}+\frac{3 l_{1}^{2} e^{-y}}{P_{r}}-\frac{S e^{-y}}{P_{r}}-\frac{2 l_{1} S e^{-y}}{P_{r}}-l_{1}^{2} S e^{-y}-l_{1} S^{2} e^{-y}+l_{1}^{3} e^{-y}+\right.
\end{aligned}
$$




$$
\begin{aligned}
& +\frac{e^{-y}}{S_{c}^{3}}-\frac{3 S e^{-y}}{S_{c}^{2}}-\frac{2 K_{r} e^{-y}}{S_{c}^{2}}+\frac{6 K_{r} S e^{-y}}{S_{c}}+\frac{3 S^{2} e^{-y}}{S_{c}}+\frac{3 K_{r}^{2} e^{-y}}{S_{c}}-\frac{K_{r} e^{-y}}{S_{c}}+ \\
& \left.-K_{r} S^{2} e^{-y}-3 K_{r}^{2} S e^{-y}-K_{r}^{3} e^{-y}\right) \frac{t^{4}}{4 !}+L^{-1}\left\{\frac { 1 } { S } L \left\{S \frac{\partial u}{\partial y}+\frac{\partial^{2} u}{\partial y^{2}}+\alpha \frac{\partial^{3} u}{\partial y^{2} \partial t}+\right.\right. \\
& +\beta_{a} \frac{\partial^{4} u}{\partial y^{2} \partial t^{2}}+\beta_{b}\left(\frac{\partial u}{\partial y}\right)^{2} \frac{\partial^{2} u}{\partial y^{2}}+\gamma_{\mathrm{a}} \frac{\partial^{5} u}{\partial y^{2} \partial t^{3}}+ \\
& \left.+\gamma_{b}\left[2 \frac{\partial u}{\partial y} \frac{\partial^{2} u}{\partial y^{2}} \frac{\partial^{2} u}{\partial t \partial y}+\left(\frac{\partial u}{\partial y}\right)^{2} \frac{\partial^{3} u}{\partial y^{2} \partial t}\right]-l_{2} u\right\} .
\end{aligned}
$$

Applying the homotopy perturbation method to Eq.(3.24), gives

$$
\begin{aligned}
& \sum_{n=0}^{\infty} P^{n} u_{n}(y, t)=\lambda+e^{-y}+\left(G_{c} e^{-y}+G_{r} e^{-y}\right) t+\left(\frac{e^{-y}}{P_{r}}-2 S e^{-y}+l_{1} e^{-y}+\frac{e^{-y}}{S_{c}}+\right. \\
& \left.-K_{r} e^{-y}\right) \frac{t^{2}}{2 !}+\left(\frac{e^{-y}}{P_{r}^{2}}+\frac{2 l_{1} e^{-y}}{P_{r}}+l_{1}^{2} e^{-y}+\frac{e^{-y}}{S_{c}^{2}}-\frac{2 S e^{-y}}{S_{c}}-\frac{2 K_{r} e^{-y}}{S_{c}}+2 K_{r} S e^{-y}+\right. \\
& \left.+K_{r}^{2} e^{-y}\right) \frac{t^{3}}{3 !}+\left(\frac{e^{-y}}{P_{r}^{3}}+\frac{3 l_{1} e^{-y}}{P_{r}^{2}}-\frac{S^{2} e^{-y}}{P_{r}}+\frac{3 l_{1}^{2} e^{-y}}{P_{r}}-\frac{S e^{-y}}{P_{r}}-\frac{2 l_{l} S e^{-y}}{P_{r}}-l_{1}^{2} S e^{-y}+\right. \\
& -l_{l} S^{2} e^{-y}+l_{l}^{3} e^{-y}+\frac{e^{-y}}{S_{c}^{3}}-\frac{3 S e^{-y}}{S_{c}^{2}}-\frac{2 K_{r} e^{-y}}{S_{c}^{2}}+\frac{6 K_{r} S e^{-y}}{S_{c}}+\frac{3 S^{2} e^{-y}}{S_{c}}+\frac{3 K_{r}^{2} e^{-y}}{S_{c}}+ \\
& \left.-\frac{K_{r} e^{-y}}{S_{c}}-K_{r} S^{2} e^{-y}-3 K_{r}^{2} S e^{-y}-K_{r}^{3} e^{-y}\right) \frac{t^{4}}{4 !}+P\left(L ^ { - 1 } \left\{\frac { 1 } { s } L \left\{S \frac{\partial u}{\partial y}+\frac{\partial^{2} u}{\partial y^{2}}+\right.\right.\right. \\
& +\alpha \frac{\partial^{3} u}{\partial y^{2} \partial t}+\beta_{\mathrm{a}} \frac{\partial^{4} u}{\partial y^{2} \partial t^{2}}+\beta_{b} H_{a}\left(u_{n}\right)+\gamma_{1} \frac{\partial^{5} u}{\partial y^{2} \partial t^{3}}+ \\
& \left.\left.\left.+\gamma_{b}\left[2 H_{b}\left(u_{n}\right)+H_{c}\left(u_{n}\right) \frac{\partial u}{\partial y} \frac{\partial^{2} u}{\partial y^{2}} \frac{\partial^{2} u}{\partial t \partial y}+\left(\frac{\partial u}{\partial y}\right)^{2} \frac{\partial^{3} u}{\partial y^{2} \partial t}\right]-l_{2} u\right\}\right\}\right)
\end{aligned}
$$

where, $H_{a}\left(u_{n}\right), H_{b}\left(u_{n}\right)$ and $H_{c}\left(u_{n}\right)$ are the He's polynomials for

$$
\left(\frac{\partial u}{\partial y}\right)^{2}, \frac{\partial u}{\partial y} \frac{\partial^{2} u}{\partial y^{2}} \frac{\partial^{2} u}{\partial t \partial y} \quad \text { and } \quad\left(\frac{\partial u}{\partial y}\right)^{2} \frac{\partial^{3} u}{\partial y^{2} \partial t}
$$

respectively.

The He's polynomials for $\left(\frac{\partial u}{\partial y}\right)^{2}$ are as follows 


$$
\left\{\begin{array}{l}
H_{0}(u)=\left(u_{0}^{\prime}\right)^{2}, \\
H_{1}(u)=2 u_{0}^{\prime} u_{1}^{\prime}, \\
H_{2}(u)=2 u_{0}^{\prime} u_{2}^{\prime}+\left(u_{1}^{\prime}\right)^{2}, \\
H_{3}(u)=2 u_{1}^{\prime} u_{2}^{\prime}, \\
\vdots
\end{array}\right.
$$

The He's polynomials for $\frac{\partial u}{\partial y} \frac{\partial^{2} u}{\partial y^{2}} \frac{\partial^{2} u}{\partial t \partial y}$ are as follows

$$
\left\{\begin{array}{l}
H_{0}(u)=u_{0}^{\prime \prime \prime} u_{0 t}^{\prime}, \\
H_{1}(u)=u_{0}^{\prime \prime \prime} u_{1 t}^{\prime}+u_{1}^{\prime \prime \prime} u_{1 t}^{\prime}, \\
H_{2}(u)=u_{0}^{\prime \prime \prime} u_{2 t}^{\prime \prime}+u_{1}^{\prime \prime \prime} u_{1 t}^{\prime \prime}+u_{2}^{\prime \prime \prime} u_{0 t}^{\prime}, \\
H_{3}(u)=u_{1}^{\prime \prime \prime} u_{2 t}^{\prime \prime}+u_{2}^{\prime \prime \prime} u_{1 t}^{\prime \prime}, \\
\vdots
\end{array}\right.
$$

The He's polynomials for $\left(\frac{\partial u}{\partial y}\right)^{2} \frac{\partial^{3} u}{\partial y^{2} \partial t}$ are as follows

$$
\left\{\begin{array}{l}
H_{0}(u)=\left(u_{0}^{\prime}\right)^{2}\left(u_{0}^{\prime \prime} u_{0 t}^{\prime}\right), \\
H_{1}(u)=\left(u_{0}^{\prime}\right)^{2}\left(u_{0}^{\prime \prime} u_{1 t}^{\prime}\right)+\left(u_{0}^{\prime}\right)^{2}\left(u_{1}^{\prime \prime} u_{0 t}^{\prime}\right)+2 u_{0}^{\prime} u_{1}^{\prime}\left(u_{0}^{\prime \prime} u_{0 t}^{\prime}\right), \\
H_{2}(u)=\left(u_{0}^{\prime}\right)^{2}\left(u_{0}^{\prime \prime} u_{2 t}^{\prime}\right)+\left(u_{0}^{\prime}\right)^{2}\left(u_{1}^{\prime \prime} u_{1 t}^{\prime}\right)+\left(u_{0}^{\prime}\right)^{2}\left(u_{2}^{\prime \prime} u_{0 t}^{\prime}\right)+ \\
+2 u_{0}^{\prime} u_{1}^{\prime}\left(u_{0}^{\prime \prime} u_{1 t}^{\prime}\right)+2 u_{0}^{\prime} u_{1}^{\prime}\left(u_{1}^{\prime \prime} u_{0 t}^{\prime}\right)+2 u_{0}^{\prime} u_{2}^{\prime}\left(u_{0}^{\prime \prime} u_{0 t}^{\prime}\right), \\
\vdots
\end{array}\right.
$$

Now, comparing the like powers of " $P$ " in Eq.(3.25) and equating their coefficients gives 


$$
\begin{aligned}
& P^{0} ; u_{0}(y, t)=\lambda+e^{-y}+\left(G_{c} e^{-y}+G_{r} e^{-y}\right) t+\left(\frac{e^{-y}}{P_{r}}-2 S e^{-y}+l_{1} e^{-y}+\frac{e^{-y}}{S_{c}}-K_{r} e^{-y}\right) \frac{t^{2}}{2 !}+ \\
& +\left(\frac{e^{-y}}{P_{r}^{2}}+\frac{2 l_{1} e^{-y}}{P_{r}}+l_{1}^{2} e^{-y}+\frac{e^{-y}}{S_{c}^{2}}-\frac{2 S e^{-y}}{S_{c}}-\frac{2 K_{r} e^{-y}}{S_{c}}+2 K_{r} S e^{-y}+K_{r}^{2} e^{-y}\right) \frac{t^{3}}{3 !}+ \\
& +\left(\frac{e^{-y}}{P_{r}^{3}}+\frac{3 l_{1} e^{-y}}{P_{r}^{2}}-\frac{S^{2} e^{-y}}{P_{r}} \frac{3 l_{1}^{2} e^{-y}}{P_{r}}-\frac{S e^{-y}}{P_{r}}-\frac{2 l_{1} S e^{-y}}{P_{r}}-l_{1}^{2} S e^{-y}-l_{1} S^{2} e^{-y}+l_{1}^{3} e^{-y}+\right. \\
& +\frac{e^{-y}}{S_{c}^{3}}-\frac{3 S e^{-y}}{S_{c}^{2}}-\frac{2 K_{r} e^{-y}}{S_{c}^{2}}+\frac{6 K_{r} S e^{-y}}{S_{c}}+\frac{3 S^{2} e^{-y}}{S_{c}}+\frac{3 K_{r}^{2} e^{-y}}{S_{c}}-\frac{K_{r} e^{-y}}{S_{c}}+ \\
& \left.-K_{r} S^{2} e^{-y}-3 K_{r}^{2} S e^{-y}-K_{r}^{3} e^{-y}\right) \frac{t^{4}}{4 !} \\
& P^{l} ; u_{1}(y, t)=L^{-1}\left\{\frac { 1 } { s } L \left\{S \frac{\partial u_{0}}{\partial y}+\frac{\partial^{2} u_{0}}{\partial y^{2}}+\alpha \frac{\partial^{3} u_{0}}{\partial y^{2} \partial t}+\beta_{a} \frac{\partial^{4} u_{0}}{\partial y^{2} \partial t^{2}}+\beta_{b}\left(u_{0}^{\prime}\right)^{2}\left(u_{0}^{\prime \prime}\right)+\right.\right. \\
& \left.\left.+\gamma_{a} \frac{\partial^{5} u_{0}}{\partial y^{2} \partial t^{3}}+\gamma_{b}\left[2 u_{0}^{\prime \prime \prime} u_{0 t}^{\prime}+\left(u_{0}^{\prime}\right)^{2}\left(u_{0}^{\prime \prime} u_{0 t}^{\prime}\right)\right]-l_{2} u_{0}\right\}\right\}
\end{aligned}
$$

or

$$
\begin{aligned}
& u_{1}(y, t)=\left(e^{-y}-S e^{-y}+\alpha G_{c} e^{-y}+\alpha G_{r} e^{-y}+\frac{\beta_{a} e^{-y}}{S_{c}}-\beta_{a} K_{r} e^{-y}+\frac{\beta_{a} e^{-y}}{P_{r}}-\beta_{b} e^{-y}+\right. \\
& +\frac{\gamma_{a} e^{-y}}{S_{c}^{3}}-\frac{2 \gamma_{a} S e^{-y}}{S_{c}}-\frac{2 \gamma_{a} K_{r} e^{-y}}{S_{c}}+2 \gamma_{a} K_{r} S e^{-y}+\gamma_{a} K_{r}^{2} e^{-y}+\frac{\gamma_{a} e^{-y}}{P_{r}^{2}}+\frac{2 \gamma_{a} l_{1} e^{-y}}{P_{r}}+ \\
& \left.+\gamma_{a} l_{1}^{2} e^{-y}-2 \gamma_{a} G_{c} e^{-2 y}+2 \gamma_{a} G_{r} e^{-2 y}+\gamma_{b} G_{c} e^{-3 y}+\gamma_{b} G_{r} e^{-3 y}-l_{2} e^{-y}-\lambda l_{2}\right) t+ \\
& +\left(G_{c} e^{-y}+G_{r} e^{-y}-G_{c} S e^{-y}-G_{r} S e^{-y}+\frac{\alpha e^{-y}}{S_{c}}-2 \alpha S e^{-y}-\frac{\alpha e^{-y}}{P_{r}}+\alpha l_{l} e^{-y}+\frac{\beta_{a} e^{-y}}{S_{c}^{2}}+\right. \\
& -\frac{2 \beta_{a} S e^{-y}}{S_{c}}-\frac{2 \beta_{a} K_{r} e^{-y}}{S_{c}}+2 \beta_{a} K_{r} S e^{-y}+\beta_{a} K_{r}^{2} e^{-y}+\frac{\beta_{a} e^{-y}}{P_{r}^{2}}-\frac{2 \beta_{a} l_{l} e^{-y}}{P_{r}}+\beta_{a} l_{l}^{2} e^{-y}+ \\
& +3 \beta_{b} G_{c} e^{-3 y}+3 \beta_{b} G_{r} e^{-3 y}+\frac{\gamma_{a} e^{-y}}{S_{c}^{3}}-\frac{3 \gamma_{a} e^{-y}}{S_{c}^{2}}-\frac{6 \gamma_{a} K_{r} S e^{-y}}{S_{c}}+\frac{6 \gamma_{a} S^{2} e^{-y}}{S_{c}}+ \\
& -6 \gamma_{a} K_{r} S^{2} e^{-y}-3 \gamma_{a} K_{r}^{2} S e^{-y}-\gamma_{a} K_{r}^{3} e^{-y}+\frac{\gamma_{a} e^{-y}}{P_{r}^{3}}+\frac{3 \gamma_{a} e^{-y}}{P_{r}^{2}}-\frac{\gamma_{a} S^{2} e^{-y}}{P_{r}}+ \\
& +\frac{3 \gamma_{a} l_{l}^{2} e^{-y}}{P_{r}}-\frac{\gamma_{a} S e^{-y}}{P_{r}}-\gamma_{a} l_{1}^{2} S e^{-y}-\gamma_{a} l_{1} S^{2} e^{-y}+\gamma_{a} l_{l}^{3} e^{-y}-2 \gamma_{b} G_{c}^{2} e^{-3 y}+ \\
& \left.-3 \gamma_{b} G_{r}^{2} e^{-3 y}-4 \gamma_{b} G_{C} G_{r} e^{-3 y}-l_{2} G_{c} e^{-y}-l_{2} G_{r} e^{-y}\right) \frac{t^{2}}{2 !}+ \\
& +\left(\frac{e^{-y}}{S_{c}}-K_{r} e^{-y}+\frac{e^{-y}}{P_{r}}+l_{l} e^{-y}-\frac{S e^{-y}}{S_{c}}+K_{r} S e^{-y}-\frac{S e^{-y}}{P_{r}}+S l_{l} e^{-y}+\frac{\alpha e^{-y}}{S_{c}^{2}}+\right.
\end{aligned}
$$




$$
\begin{aligned}
& -\frac{2 \alpha S e^{-y}}{S_{c}}-\frac{2 \alpha K_{r} e^{-y}}{S_{c}}+2 \alpha K_{r} S e^{-y}+\alpha K_{r}^{2} e^{-y}+\frac{\alpha e^{-y}}{P_{r}^{2}}+\frac{2 \alpha l_{1} e^{-y}}{P_{r}}+\alpha l_{l}^{2} e^{-y}+ \\
& +\frac{\beta_{a} e^{-y}}{S_{c}^{3}}-\frac{3 \beta_{a} S e^{-y}}{S_{c}^{2}}-\frac{2 \beta_{a} K_{r} e^{-y}}{S_{c}^{2}}+\frac{6 \beta_{a} K_{r} S e^{-y}}{S_{c}}+\frac{3 \beta_{a} S^{2} e^{-y}}{S_{c}}+\frac{3 \beta_{a} K_{r}^{2} e^{-y}}{S_{c}}+ \\
& -\frac{\beta_{a} K_{r} e^{-y}}{S_{c}}-3 \beta_{a} K_{r} S^{2} e^{-y}-3 \beta_{a} K_{r}^{2} S e^{-y}-\beta_{a} K_{r}^{3} e^{-y}+\frac{\beta_{a} e^{-y}}{P_{r}^{3}}+\frac{3 \beta_{a} l_{l} e^{-y}}{P_{r}^{2}}+ \\
& -\frac{\beta_{a} S^{2} e^{-y}}{P_{r}}+\frac{3 \beta_{a} l_{l}^{2} e^{-y}}{P_{r}}-\frac{\beta_{a} S e^{-y}}{P_{r}}-\frac{2 \beta_{a} l_{l} S e^{-y}}{P_{r}}-\beta_{a} l_{l}^{2} S e^{-y}-\beta_{a} l_{l} S^{2} e^{-y}+ \\
& +\beta_{a} l_{l}^{3} e^{-y}+\beta_{a} S^{3} e^{-y}+12 \beta_{b} G_{c} G_{r} e^{-3 y}-6 \beta_{b} G_{c}^{2} e^{-3 y}-6 \beta_{b} G_{r}^{2} e^{-3 y}+ \\
& -\frac{l_{1} e^{-y}}{S_{c}}+l_{2} K_{r} e^{-y}-\frac{l_{2} e^{-y}}{P_{r}}+6 \gamma_{b} G_{c}^{2} G_{r} e^{-3 y}+6 \gamma_{b} G_{c} G_{r}^{2} e^{-3 y}+2 \gamma_{b} G_{c}^{3} e^{-3 y}+ \\
& +2 \gamma_{b} G_{r}^{3} e^{-3 y}-l_{1} l_{2} e^{-y}-\frac{l_{2} e^{-y}}{P_{r}}+6 \gamma_{b} G_{c}^{2} G_{r} e^{-3 y}+6 \gamma_{b} G_{c} G_{r}^{2} e^{-3 y}+2 \gamma_{b} G_{c}^{3} e^{-3 y}+ \\
& \left.+2 \gamma_{b} G_{r}^{3} e^{-3 y}-l_{l} l_{2} e^{-y}\right) \frac{t^{3}}{3 !}+\left(\frac{S e^{-y}}{S_{c}^{2}}+\frac{2 S^{2} e^{-y}}{S_{c}}+\frac{2 K_{r} S e^{-y}}{S_{c}}-2 K_{r} S^{2} e^{-y}-K_{r}^{2} S e^{-y}\right. \\
& -\frac{S e^{-y}}{P_{r}^{2}}-\frac{2 l_{l} S e^{-y}}{P_{r}}-l_{1}^{2} S e^{-y}+\frac{e^{-y}}{S_{c}^{2}}-\frac{2 S e^{-y}}{S_{c}}-\frac{2 K_{r} e^{-y}}{S_{c}}+2 K_{r} S e^{-y}+K_{r}^{2} e^{-y}+ \\
& +\frac{e^{-y}}{P_{r}^{2}}+\frac{2 l_{1} e^{-y}}{P_{r}}+l_{l}^{2} e^{-y}+\frac{\alpha e^{-y}}{S_{c}^{3}}-\frac{3 \alpha S e^{-y}}{S_{c}^{2}}-\frac{2 \alpha K_{r} e^{-y}}{S_{c}^{2}}+\frac{6 \alpha K_{r} S e^{-y}}{S_{c}}+\frac{3 \alpha S^{2} e^{-y}}{S_{c}}+ \\
& +\frac{3 \alpha K_{r}^{2} e^{-y}}{S_{c}}-\frac{\alpha K_{r} e^{-y}}{S_{c}}-3 \alpha K_{r} S^{2} e^{-y}-3 \alpha K_{r}^{2} S e^{-y}-\alpha K_{r}^{3} e^{-y}+\frac{\alpha e^{-y}}{P_{r}^{3}}+\frac{3 \alpha l_{1} e^{-y}}{P_{r}^{2}}+ \\
& -\frac{\alpha S^{2} e^{-y}}{P_{r}}+\frac{3 \alpha l_{1}^{2} e^{-y}}{P_{r}}-\frac{\alpha S e^{-y}}{P_{r}}-\frac{\alpha l_{1} S e^{-y}}{P_{r}}-\alpha l_{1}^{2} S e^{-y}-\alpha l_{l} S^{2} e^{-y}+\alpha l_{l}^{3} e^{-y}+ \\
& +6 \beta_{b} G_{c}^{3} e^{-3 y}+18 \beta_{b} G_{c} G_{r}^{2} e^{-3 y}+6 \beta_{b} G_{r}^{3} e^{-3 y}-\frac{l_{2} e^{-y}}{S_{c}^{2}}+\frac{l_{2} S e^{-y}}{S_{c}}+\frac{2 l_{2} K_{r} e^{-y}}{S_{c}}+ \\
& \left.-2 l_{2} K_{r} S e^{-y}-l_{2} K_{r}^{2} e^{-y}-\frac{l_{2} e^{-y}}{P_{r}}-\frac{2 l_{1} l_{2} e^{-y}}{P_{r}}-l_{1}^{2} l_{2} e^{-y}\right) \frac{t^{4}}{4 !}+\left(\frac{e^{-y}}{S_{c}^{3}}-\frac{3 S e^{-y}}{S_{c}^{2}}+\right. \\
& -\frac{2 K_{r} e^{-y}}{S_{c}^{2}}+\frac{6 K_{r} S e^{-y}}{S_{c}}+\frac{3 S^{3} e^{-y}}{S_{c}}+\frac{3 K_{r}^{3} e^{-y}}{S_{c}}-\frac{K_{r} e^{-y}}{S_{c}}-3 K_{r} S^{2} e^{-y}-3 K_{r}^{2} S e^{-y} \\
& -K_{r}^{3} e^{-y}+\frac{e^{-y}}{P_{r}^{3}}+\frac{3 l_{1} e^{-y}}{P_{r}^{2}}-\frac{S^{2} e^{-y}}{P_{r}}+\frac{3 l_{1}^{2} e^{-y}}{P_{r}}-\frac{S e^{-y}}{P_{r}}-\frac{2 S l_{1} e^{-y}}{P_{r}}-l_{1}^{2} S e^{-y}-l_{1} S^{2} e^{-y}+ \\
& +l_{1}^{3} e^{-y}-\frac{S e^{-y}}{S_{c}^{3}}+\frac{3 s^{2} e^{-y}}{S_{c}^{2}}+\frac{2 K_{r} S e^{-y}}{S_{c}^{2}}-\frac{6 K_{r} S^{2} e^{-y}}{S_{c}}-\frac{3 s^{3} e^{-y}}{S_{c}}-\frac{3 K_{r}^{2} S e^{-y}}{S_{c}}+ \\
& +\frac{K_{r} S e^{-y}}{S_{c}}+3 K_{r} S^{3} e^{-y}+3 K_{r}^{2} S^{2} e^{-y}+K_{r}^{3} S e^{-y}-\frac{S e^{-y}}{P_{r}^{3}}-\frac{3 l_{l} S e^{-y}}{P_{r}^{2}}+\frac{s^{3} e^{-y}}{P_{r}}+
\end{aligned}
$$




$$
\begin{aligned}
& -\frac{3 l_{1}^{2} S e^{-y}}{P_{r}}+\frac{S^{2} e^{-y}}{P_{r}}+\frac{2 l_{1} S^{2} e^{-y}}{P_{r}}+l_{1}^{2} S^{2} e^{-y}-l_{1} S^{3} e^{-y}-l_{1}^{3} S e^{-y}-\frac{l_{2} e^{-y}}{S_{c}^{3}}+\frac{3 l_{2} S e^{-y}}{S_{c}^{2}}+ \\
& +\frac{2 l_{2} K_{r} e^{-y}}{S_{c}^{2}}-\frac{6 l_{2} K_{r} S e^{-y}}{S_{c}}-\frac{3 l_{2} S^{2} e^{-y}}{S_{c}}-\frac{3 l_{2} K_{r}^{2} e^{-y}}{S_{c}}+\frac{l_{2} K_{r} e^{-y}}{S_{c}}+3 l_{2} K_{r}^{2} S e^{-y}+ \\
& +3 l_{2} K_{r} S^{2} e^{-y}+l_{2} K_{r}^{3} e^{-y}-\frac{l_{2} e^{-y}}{P_{r}^{3}}-\frac{3 l_{1} l_{2} e^{-y}}{P_{r}^{2}}+\frac{l_{2} S^{2} e^{-y}}{P_{r}}-\frac{l_{2} S e^{-y}}{P_{r}}+\frac{2 l_{1} l_{2} S e^{-y}}{P_{r}}+ \\
& \left.+l_{1}^{2} l_{2} S e^{-y}+l_{1} l_{2} S^{2} e^{-y}-l_{1}^{3} l_{2} e^{-y}\right) \frac{t^{5}}{5 !} .
\end{aligned}
$$

Therefore, the solution to Eq.(2.16) is;

$$
u(y, t)=u_{0}(y, t)+u_{l}(y, t)+\cdots
$$

where, $u_{0}(y, t)$ and $u_{l}(y, t)$ are defined in Eq.(3.29) and (3.31) respectively.

The physical momentum, heat and mass properties such as the skin friction, Nusselt and Sherwood number, which are elucidated in Arifuzzaman et al. [8] are:

$$
\left\{\begin{array}{c}
C_{f}=-\frac{1}{2 \sqrt{2}} G_{r}^{-3 / 4}\left(\frac{\partial u}{\partial y}\right)_{y=0} \\
N_{u}=\frac{1}{2 \sqrt{2}} G_{r}^{-3 / 4}\left(\frac{\partial \theta}{\partial y}\right)_{y=0}, \\
S_{h}=-\frac{1}{2 \sqrt{2}} G_{r}^{-3 / 4}\left(\frac{\partial C}{\partial y}\right)_{y=0}
\end{array}\right.
$$

\section{Results and discussion}

An unsteady MHD flow of a fourth-grade fluid in a horizontal parallel plates channel with thermal radiation, chemical reaction and suction effects has been analyzed. The impact of thermal radiation, chemical reaction, suction, third and fourth-grade parameters along with other pertinent flow parameters are plotted graphically on different flow fields. The default values for the pertinent flow parameters are taken as (Arifuzzaman [8])

$$
\begin{aligned}
& \lambda=0.30, \quad \alpha=0.20, \quad \beta_{a}=0.05, \quad \beta_{b}=0.05, \quad \gamma_{a}=0.05, \quad \gamma_{b}=0.0 .05, \quad S_{c}=0.50, \\
& G_{r}=5, \quad G_{c}=5, \quad P_{r}=0.71, \quad H a=0.30, \quad \delta=.05, \quad D a=1.00, \quad K_{r}=0.50 .
\end{aligned}
$$


To validate the present work, a numerical comparison is provided in Tab.1. It can be seen there is no significant difference. The impact of flow parameters on the skin friction $C_{f}$, Nusselt number $N_{u}$, Sherwood number $S_{h}$ is also investigated and presented in Tab.2.

Table 1. Validation of present study against Arifuzzaman [8] for the steady state Nusselt number, where $P_{r}=0.71$.

\begin{tabular}{|cccc|}
\hline$\delta$ & $\begin{array}{c}\text { Nusselt number } N_{u} \\
\text { (Present work) }\end{array}$ & $\begin{array}{c}\text { Nusselt number } N_{u} \\
\text { (Arifuzzaman } \text { et al. } \text { [8]) }\end{array}$ & Difference \\
\hline 0.05 & 0.54190 & 0.49308 & 0.05 \\
0.10 & $0 . .63411$ & 0.58207 & 0.05 \\
0.15 & 0.40762 & 0.39764 & 0.01 \\
0.20 & 0.40981 & 0.37984 & 0.03 \\
\hline
\end{tabular}

Table 2. Computational values of the skin friction $C_{f}$, Nusselt number $N_{u}$ and Sherwood number $S_{h}$.

\begin{tabular}{|c|c|c|c|c|c|c|c|c|c|c|c|}
\hline$s$ & $\delta$ & $H a$ & $P_{r}$ & $\alpha$ & $\beta_{a}=\beta_{b}$ & $\gamma_{a}=\gamma_{b}$ & $K_{r}$ & $S_{c}$ & $C_{f}$ & $N_{u}$ & $S_{h}$ \\
\hline 0.20 & 0.05 & 0.30 & 0.71 & 0.05 & 0.05 & 0.05 & 0.50 & 0.50 & 2.0045 & 0.5519 & 0.3494 \\
\hline 0.30 & & & & & & & & & 1.9544 & 0.5383 & 0.3401 \\
\hline 0.40 & 0.10 & & & & & & & & 1.9092 & 0.5358 & 0.3312 \\
\hline 0.50 & 0.15 & & & & & & & & 1.8639 & 0.5328 & 0.3227 \\
\hline & 0.20 & 0.60 & & & & & & & 1.8959 & 0.5885 & 0.3494 \\
\hline & & 0.80 & & & & & & & 1.7986 & 0.5519 & 0.3494 \\
\hline & & 1.00 & 1.00 & & & & & & 1.6727 & 0.5510 & 0.3494 \\
\hline & & & 1.50 & & & & & & 1.9283 & 0.6102 & 0.3494 \\
\hline & & & 2.00 & 0.10 & 0.10 & & & & 2.2777 & 0.7171 & 0.3494 \\
\hline 0.80 & & & & 0.20 & 0.20 & & & & 2.8109 & 0.4615 & 0.2996 \\
\hline & & & & 0.30 & 0.30 & 0.10 & & & 3.3347 & 0.5519 & 0.3494 \\
\hline & & & & & & 0.20 & & & 3.8775 & 0.5519 & 0.3494 \\
\hline & & & & & & 0.30 & 1.00 & & 4.2177 & 0.5519 & 0.3064 \\
\hline & & & & & & & 1.50 & 0.70 & 1.9216 & 0.5519 & 0.2034 \\
\hline & & & & & & & 2.00 & 0.80 & 1.9021 & 0.5519 & 0.1493 \\
\hline & & & & & & & & 1.20 & 1.9107 & 0.5519 & 0.0911 \\
\hline
\end{tabular}

Table 2 presented the effect of flow parameters on the skin friction $C_{f}$, Nusselt number $N_{u}$, Sherwood number $S_{h}$. It is seen that the skin friction develops due to the increase in $\delta, P_{r}, \alpha, \beta_{a}, \beta_{b}, \gamma_{a}$ and $\gamma_{b}$ but diminishes due to an increase in $S, H a, K_{r}$ and $S_{c}$. The Nusselt number increases with an increase of $P_{r}$ and decreases with an increase in $S$ and $\delta$. The Sherwood number diminishes due an increase in $S, K_{r}$ and $S_{c}$. Figures 2 and 3 depict the velocity and temperature fields for the increment in the thermal radiation parameter $\delta(0.05 \leq \delta \geq 0.40)$. Thermal radiation is known as electromagnetic radiation or the conversion of thermal energy which generates the thermal motion of particles in matter. Thermal radiation could be attributed to thermal excitation. Both velocity and temperature fields are affected significantly by on increase in the thermal radiation parameter $(\delta)$. 


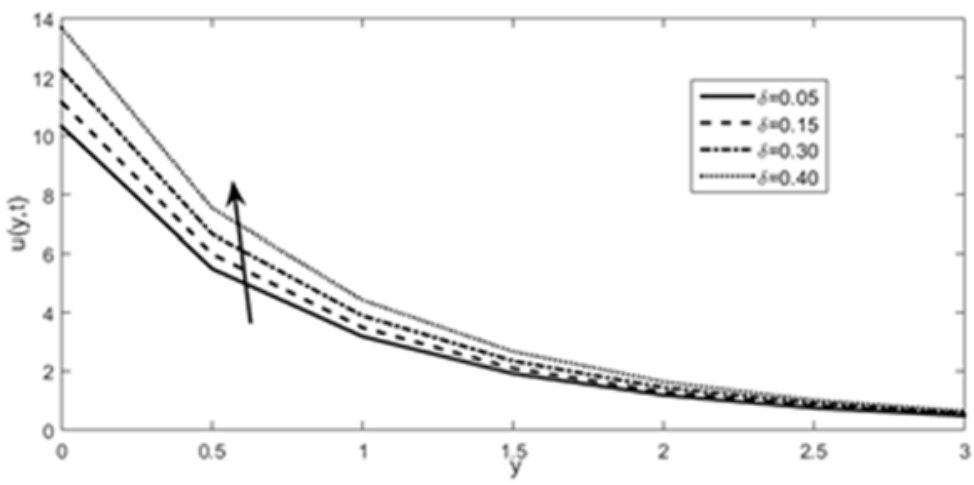

Fig.2. Effect of $\delta$ on Velocity profile $u$.

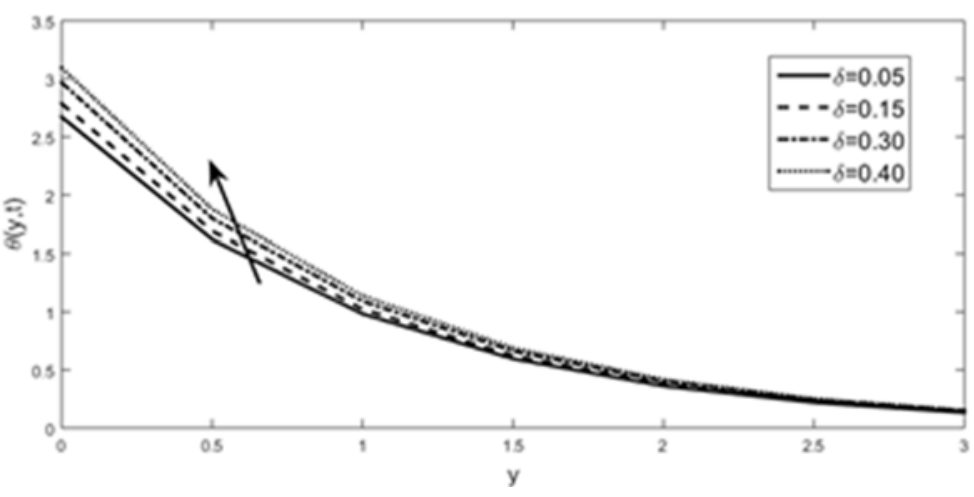

Fig.3. Effect of $\delta$ on temperature distribution $\theta$.

The effect of the chemical reaction parameter $\left(K_{r}\right)$ on velocity and concentration profiles is depicted in Figs 4 and 5 respectively. Due to the rise of the chemical reaction $\left(K_{r}\right)$ from $0.50 \leq K_{r} \geq 2.00$, the velocity field decreases, and the concentration field also decreases. Physically, chemical reaction occurs with more disturbance which develops the molecular motion and upsurges the heat transport phenomena and as a result retards the velocity of the flow.

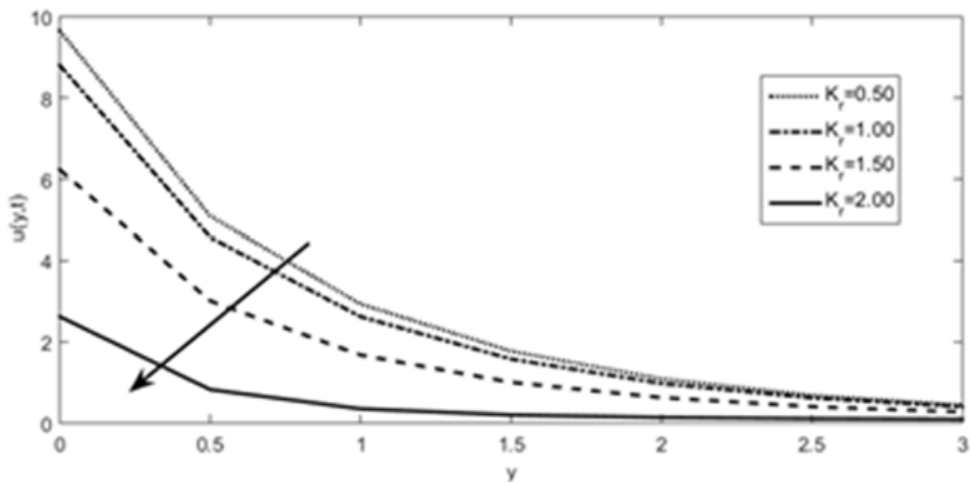

Fig.4. Effect of $K_{r}$ on velocity profile $u$. 


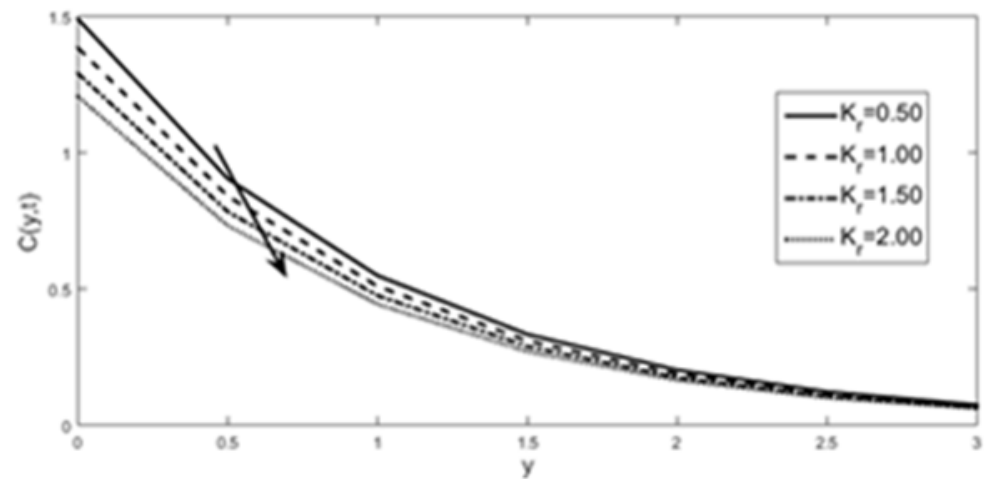

Fig.5. Effect of $K_{r}$ on concentration distribution $C$.

Figure 6 illustrates the drag force effect on the fluid flow. The velocity profile decreases with an increment in the Hartmann number $(0.10 \leq H a \geq 0.90)$. The role of the Hartmann number, which is the magnetic parameter, is to suppress turbulence. Physically, when a magnetic field is applied to any fluid, the apparent viscosity of the fluid increases to the point of becoming a viscous elastic solid. It is of great interest that yield stress of the fluid can be controlled very accurately through variation of the magnetic field intensity. The result is that the ability of the fluid to transmit force can be controlled with the help of an electromagnet which give rise to many possible control-based applications, including MHD power generation, electromagnetic casting of metals, MHD propulsion, etc.

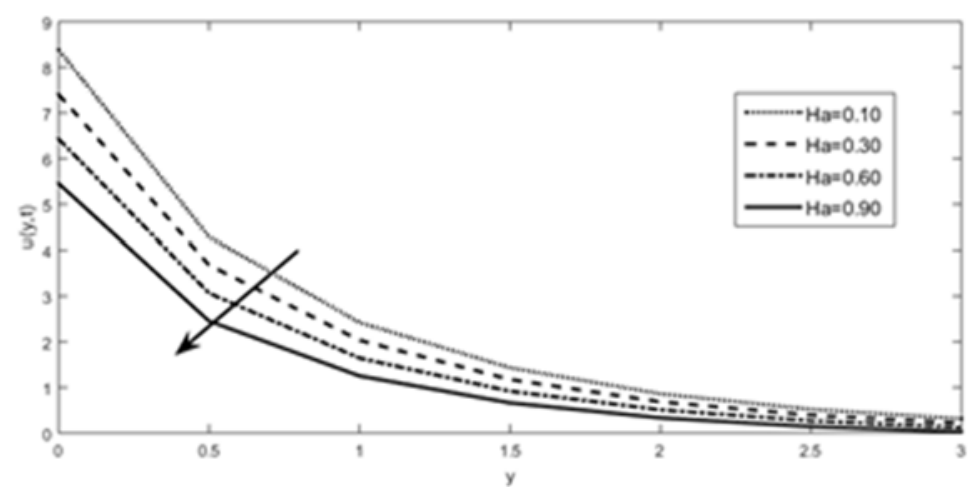

Fig.6. Effect of $H a$ on velocity profile $u$.

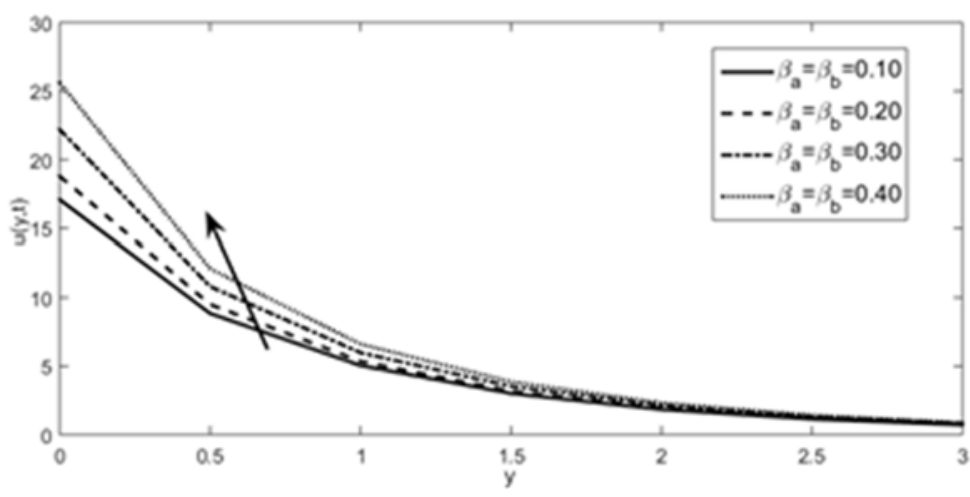

Fig.7. Effect of $\beta_{a}$ and $\beta_{b}$ on velocity profile $u$. 
The effect of third-grade and fourth-grade parameters on velocity profiles are respectively illustrated in Figs 7 and 8. It is observed that the velocity profile develops with an increase in both third-grade $\left(0.10 \leq\left(\beta_{a}=\beta_{b}\right) \geq 0.40\right)$ and fourth-grade $\left(0.10 \leq\left(\gamma_{a}=\gamma_{b}\right) \geq 0.40\right)$ parameters.

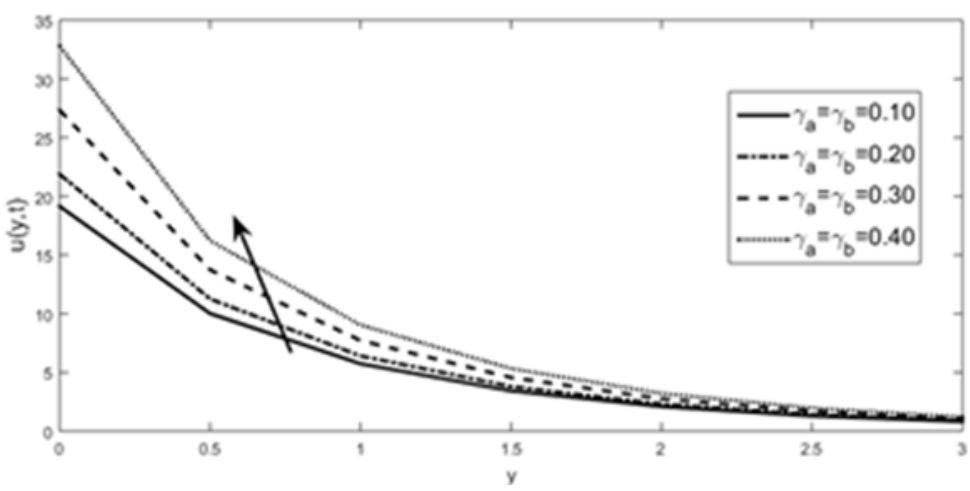

Fig.8. Effect of $\gamma_{a}$ and $\gamma_{b}$ on velocity profile $u$.

The impact of the suction parameter $S$ on velocity, temperature and concentration profiles is illustrated in Figs 9, 10 and 11 respectively. It is clearly seen that velocity, temperature and concentration profiles diminish with an increase of $S(0.10 \leq S \geq 0.40)$. This is due to the porosity of plates.

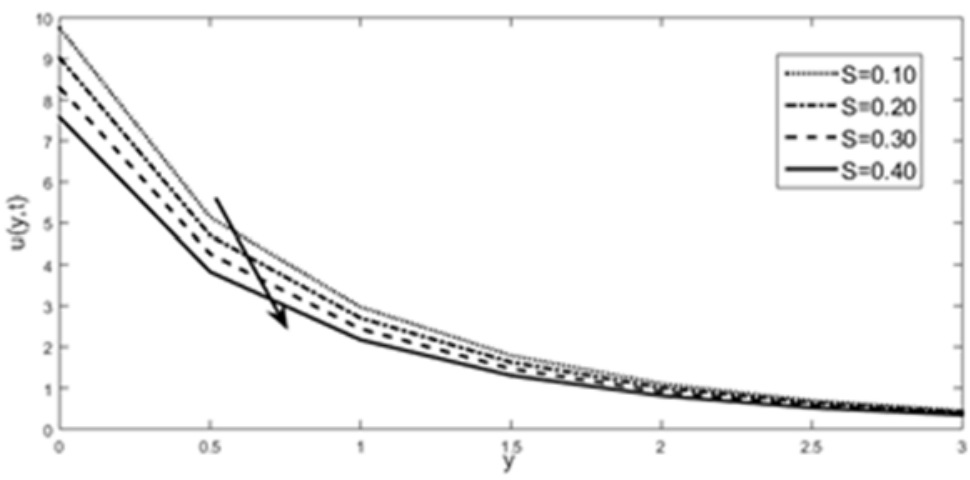

Fig.9. Effect of $S$ on velocity profile $u$.

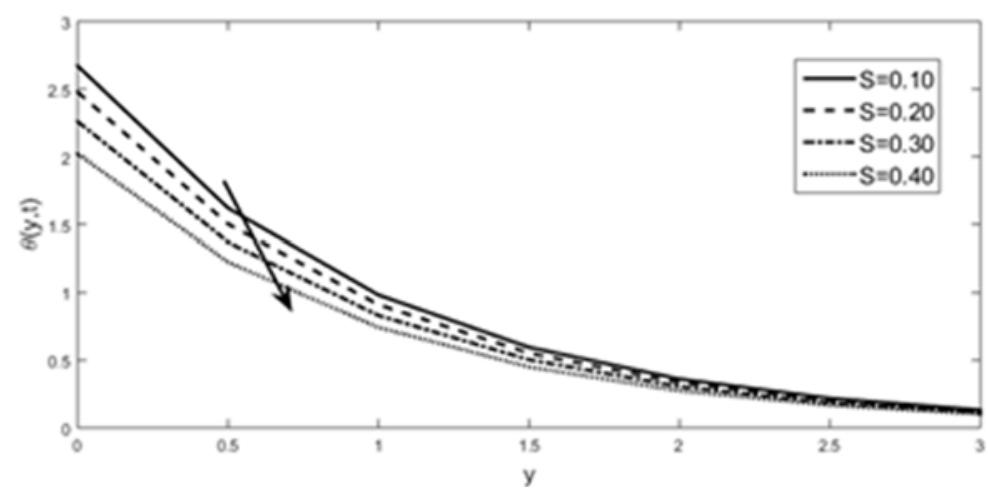

Fig.10. Effect of $S$ on temperature distribution $\theta$. 


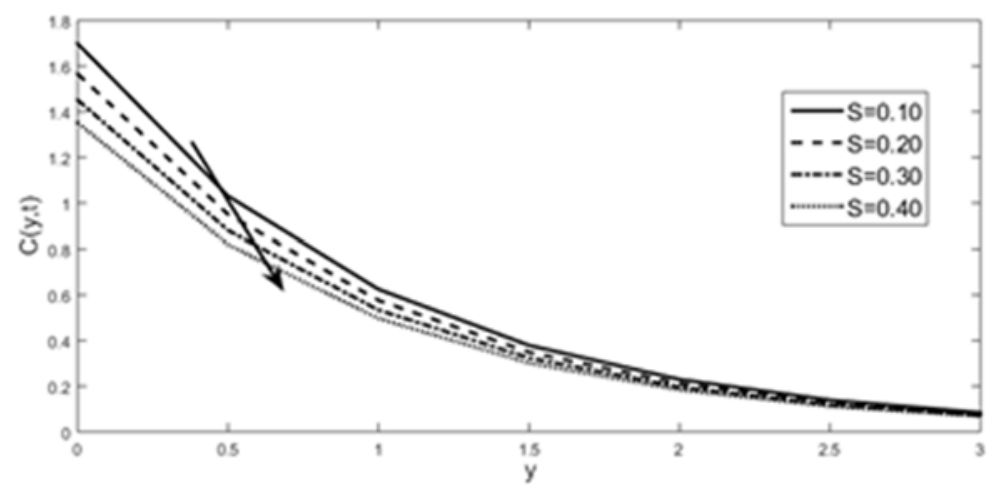

Fig.11. Effect of $S$ on concentration distribution $\theta$.

\section{Conclusion}

An unsteady MHD flow of fourth-grade in a horizontal parallel plates channel with thermal radiation, chemical reaction and suction effects has been investigated. The solutions for the nonlinear partial differential equations are obtained by the He-Laplace scheme. The effects of flow parameters on velocity, temperature and concentration profiles are depicted in figures and discussed. From the results obtained, the findings are:

- Velocity and temperature fields rise due to the increment the thermal radiation parameter.

- For upsurging data of chemical reaction, velocity and concentration fields diminish.

- Velocity, temperature and concentration fields diminish due to the increment in the suction parameter.

- Velocity profile goes up when third and fourth-grade parameters get to rise.

- Velocity and skin friction fields decline due to the increment in the magnetic parameter.

- Nusselt number distribution rises due to the enhancement in the thermal radiation parameter and drops due to an increase in the suction parameter.

- Large values of thermal radiation parameter, Prandtl number, second, third and fourth-grade parameters increase the skin friction while higher values of the Schmidt number, chemical reaction and suction parameters diminish the skin friction.

- The rate of mass transfer diminishes due to the rise in the Schmidt number, chemical reaction parameter and suction parameter.

The results of this work are applicable in many industrial production processes such as in the drilling of oil and gas wells, polymer extrusion from dye, glass fiber, paper production and draining of plastics films, etc.

This work can be extended to a plane Poisueille flow of a fourth grade fluid by taking into account the viscous dissipation and buoyancy effects.

\section{Acknowledgements}

The authors gratefully acknowledge the assistance and contributions at Professor Amos Sesan Idowu from the Department of Mathematics, University of Ilorin, Nigeria and Professor Simon Daniel from the Department of Mathematical Sciences, Kaduna State University, Nigeria to improve the manuscript.

\section{Nomenclature}

$$
\begin{aligned}
B_{0} & - \text { external magnetic field } \\
C & - \text { species concentration } \\
C_{p} & - \text { specific heat capacity } \\
C_{f} & - \text { skin friction }
\end{aligned}
$$




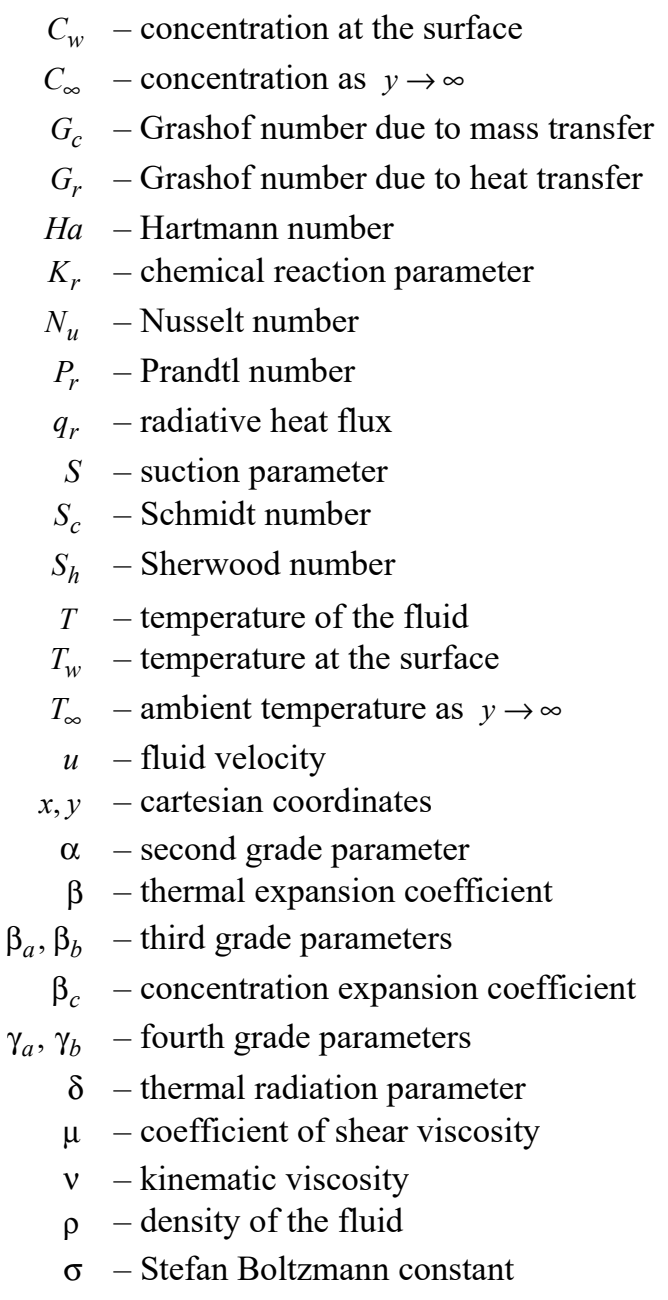

\section{References}

[1] Rehan A.S., Islam S. and Siddiqui A.M. (2010): Couette and Poiseuille flows for fourth-grade fluids using optimal homotopy asymptotic method.- World Applied Science Journal, vol.9, No.1, pp.1228-1236.

[2] Islam S., Bano Z., Siddique I. and Siddiqui A.M. (2011): The optimal solutions for the flow of a fourth-grade fluid with partial slip.- Computer and Mathematics with Application, vol.6, pp.1507-1516. Doi: 10.1016/j.camwa.2011.01.04.

[3] Shehzad N., Zeeshan A. and Ellahi R. (2018): Electroosmotic flow of MHD power law Al2O3-PVC nanofluid in a horizontal channel: Couette-Poiseuille flow model.- Commun. Theor. Phys., vol.6, No.6, pp.655-666. Doi: http://www.iopscience.iop.org/ctp.

[4] Fenuga O.J., Aroloye S.J. and Salawu S.O. (2020): Mathematical model and solution for an unsteady MHD fourth grade fluid flow over a vertical plate in a porous medium with magnetic field and suction/injection effects.- Journal of Scientific Research, vol.12, No.4, pp.485-498.

[5] Khan Z., Khan I., Ullah M. and Tlili I. (2018): Effect of thermal radiation and chemical reaction on non-Newtonian fluid through a vertically stretching porous plate with suction.- Results in Physics, vol.9, pp.1086-1095.

[6] Santhosa B., Younus S., Kamala G. and Ramana Murthy MV. (2017): Radiation and chemical effects on MHD free convective heat and mass transfer flow of a viscoelastic fluid past a porous plate with heat generation/absorption.Int J Chem Sci., vol.15, No.3, p.170

[7] Yurusoy M. (2020): Investigation of velocity profile in time dependent boundary layer flow of a modified power-law fluid of fourth grade.- Int. J. Applied Mechanics and Engineering, vol. 25, No.2, pp.176-191. 
[8] Taza G., Fazle G., Islam S., Shah R.A., Khan I., Nasir S. and Sharidan S. (2016): Unsteady thin film flow of a fourthgrade fluid over a vertical moving and oscillating belt.- Propulsion and Power Research, vol.5, No.3, pp.223-235. https://doi.org/10.1016/j.jppr.2016.07.002.

[9] Hayat T., Ellahi R. and Mahomed F.M. (2007): Exact solutions for Couette and Poiseuille flows for fourth grade fluids.- Acta Mechanica, vol.188, pp.69-78.

[10] Arifuzzaman S.M, Shakhaoth Khan Md., Al-Mamum A., Reza-E-Rabbi S.K, Biswas P. and Karim I. (2018): Hydrodynamic stability and heat and mass transfer flow analysis of MHD radiative fourth-grade fluid through porous plate with chemical reaction.- Journal of King Saud University, vol.31, No.4, pp.1388-1398. https://doi.org/10.1016/j.jksus.2018.12.009.

[11] Priyadarsan K.P. and Panda S. (2020): Heat transfer in unsteady magnetohydrodynamic flow of fourth-grade fluid through a porous medium between two infinite parallel plates with time dependent suction.- Special Topics \& Reviews in Porous Media - An International Journal, vol.11, No.3, pp.239-258.

[12] Idowu A.S. and Sani U. (2019): Thermal radiation and chemical reaction effects on unsteady magnetohydrodynamic third-grade fluid flow between stationary and oscillating plates.- Int. J. of Applied Mechanics and Engineering, vol.24, pp.269-293. doi: 10.2478/ijame-2019-0018.

[13] Coleman B.D. and Noll W. (1960): An approximation theorem for functionals with applications in continuum mechanics.- Arch. Ration. Mech. Analy., vol.6, pp.355-370

[14] Hayat T., Wang Y. and Hutter K. (2002): Flow of a fourth-grade fluid.- Math. Models Methods Appl. Sci., vol.12, pp.797-811. https://doi.org/10.1142/s0218202502001908.

[15] Hayat T., Khan M., Ayub M. and Siddiqui A.M. (2005): The unsteady Couette flow of a second grade fluid in a layer of porous medium.- Archives of Mechanics, vol.57, pp.405-416.

Received: April 1,2021

Revised: September 7, 2021 